\title{
On the helicity characteristics and induced emf of magnetic-Coriolis wave packets
}

\author{
B. R. McDermott $^{1}$ and $\quad$ P. A. Davidson $^{1}$ \\ ${ }^{1}$ Department of Engineering, University of Cambridge, Cambridge, CB2 1PZ, UK
}

\section{SUMMARY}

In a rapidly rotating Boussinesq fluid, buoyant anomalies radiate low-frequency inertial wave packets which disperse along the rotation axis. The wave packets lead to axially elongated vortices, which propagate negative (positive) kinetic helicity upwards (downwards) with respect to the rotation vector. The kinetic helicity carried by the inertial wave packets is near-maximal, relative to the velocity and vorticity fields. In classical mean-field theory, kinetic helicity is often associated with the $\alpha$-effect, which is thought to be an important ingredient for planetary dynamos. The modification of inertial wave packets in the presence of a transverse uniform magnetic field is investigated here, motivated by small-scale dynamics in planetary cores, where a large-scale magnetic field affects fluid motions. We study numerically the dispersion of wave packets from an isolated buoyant source and from a random layer of buoyant anomalies, while varying the Lehnert number $L e$ - the ratio of the frequencies of Alfvén and inertial waves. We find that for $L e<0.1$, the vortices are columnar and continue to segregate kinetic helicity so that it is negative (positive) above (below) the buoyant source. Importantly, the wave packets induce an $\alpha$-effect, which remains strong and coherent for Earth-like values of the Lehnert number $(L e<0.1)$. The interaction of wave packets emitted by multiple neighbouring buoyant sources results in an $\alpha$-effect that is stronger than the $\alpha$-effect induced by wave packets launched from an isolated buoyant source, and we provide an analytical explanation for this. The coherence of the $\alpha$-effect induced by the wave packets, for Earth-like values of the Lehnert number, lends support to the $\alpha^{2}$ dynamo model driven by helical waves. 
Key words: Core, Planetary interiors, Dynamo: theories and simulations, Geomagnetic induction

\section{INTRODUCTION}

For the liquid-metal convection in Earth's outer core, global rotation dominates the fluid motion. Through the Coriolis acceleration, the effects of rotation have strong consequences for all but the smallest scales of motion. Of secondary importance are the magnetic tension forces on the fluid, resulting from the large-scale field which is sustained by stirring motions in the electrically conducting fluid. These motions originate from the secular cooling of the Earth, and from buoyant parcels of fluid that migrate from the inner core to the mantle. It is therefore important to study the dynamics of buoyant blobs under the influence of rapid rotation and a large-scale magnetic field.

Of the numerical simulations of planetary dynamos, those which generate a predominantly dipolar magnetic field exhibit a velocity field that is composed of filamentary vortices aligned with the rotation axis. These vortices carry large amounts of kinetic helicity, which is usually distributed negative (positive) in the northern (southern) hemisphere. This anti-symmetric kinetic helicity distribution is known to be highly beneficial for dynamo action (Moffatt 1978). In addition to the columnar nature of the fluid flow, the more recent simulations have a highly time dependent buoyancy field. This observation suggests that the columnarity of the flow must be continually re-established by some short time-scale dynamics (relative to the advection time). The most robust candidate in the purely hydrodynamic case is the spontaneous emission of inertial waves by buoyant anomalies (Davidson 2014). Indeed, inertial waves have recently been identified in a moderately supercritical spherical dynamo simulation (Ranjan et al. 2018).

Inertial waves are helical waves, and this is also true of their magnetically influenced counterparts (Moffatt 1978). Such waves influenced by the magnetic field have long received attention in the dynamo community for their possible connection to the secular variation of the observed field (Hide 1966). Moffatt (1970) first noticed the potential importance of inertial waves for dynamo action. He noted that if there was a net wave-flux parallel (or anti-parallel) to the rotation axis of the planet, the mean generated electromotive force (emf) could sustain a large-scale dynamo. In 


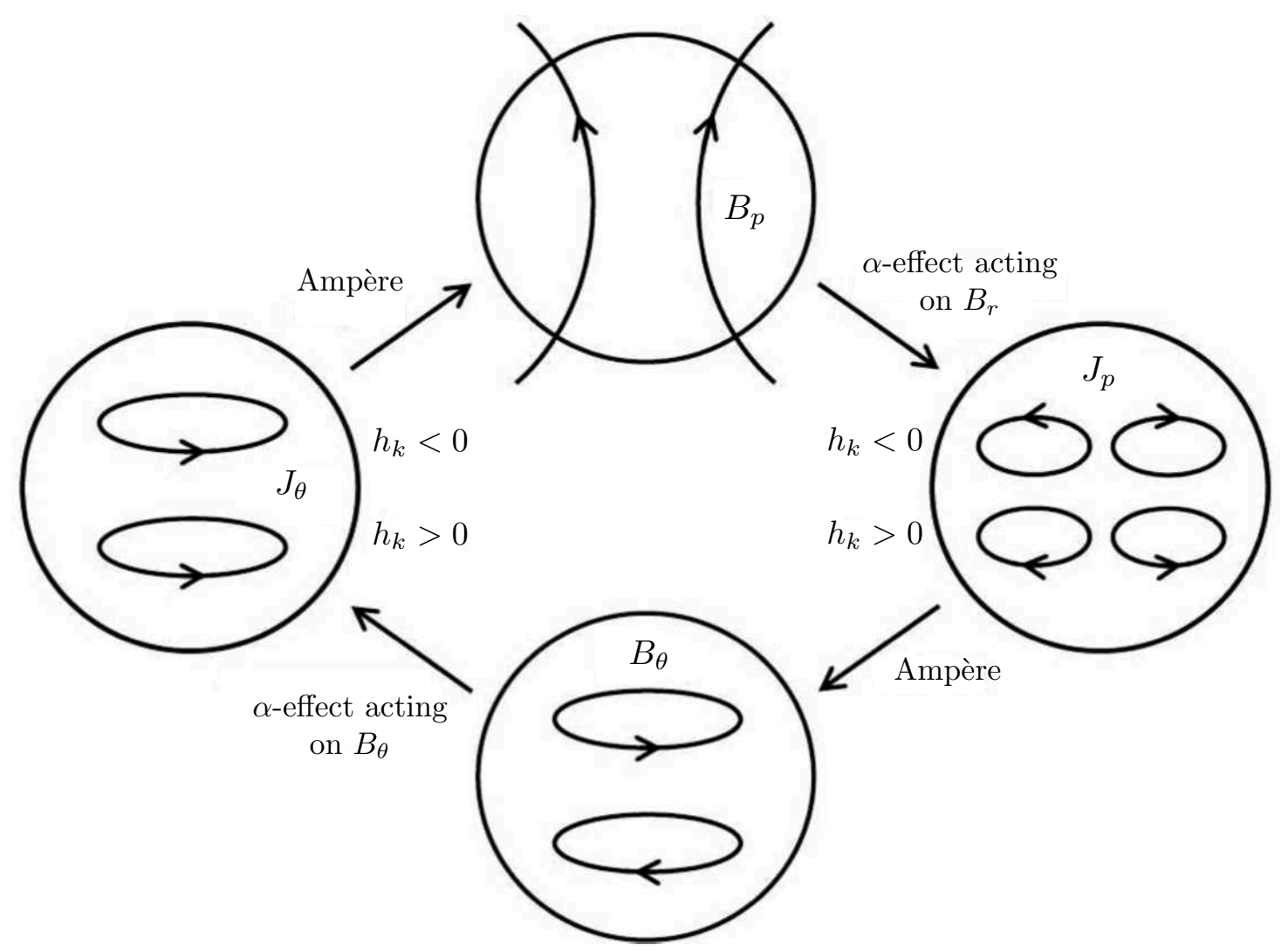

Figure 1. An $\alpha^{2}$ dynamo cartoon with net flow helicity negative (positive) in the northern (southern) hemisphere; adapted from Davidson \& Ranjan (2015).

the parlance of mean-field theory, the helical waves generate an $\alpha$-effect which sets up an $\alpha^{2}$ dynamo cycle like the one shown in Figure 1 (Davidson \& Ranjan 2015). In this schematic, $B$ is the magnetic field, $J$ is the current density, the subscripts $p, \theta$ and $r$ correspond to poloidal, azimuthal and cylindrical radial components, $h_{k}$ is the helicity of the flow and 'Ampère' signifies the use of Ampère's law.

The $\alpha^{2}$ dynamo cartoon rests on the assumption that the flow helicity is sufficiently intense, and that it is distributed negative (positive) in the north (south) outside the tangent cylinder (the imaginary axial cylinder which is tangent to the inner core equator). This type of helicity distribution is observed in many dynamo simulations to date, and can arise from two main mechanisms. The first mechanism is Ekman pumping at the outer boundary, which prevails in the more viscous simulations (Roberts \& King 2013). The second is the preferential generation of inertial wave packets in the equatorial regions (Davidson \& Ranjan 2015), such an equatorial bias in the buoyant forcing having been noted by many authors over the past two decades (Olson et al. 1999; Sakuraba \& 
Roberts 2009; Schaeffer et al. 2017). The latter is an attractive mechanism for the planets, as it is independent of viscosity and the presence of a solid outer boundary.

Davidson \& Ranjan (2015) showed that it is indeed feasible for a planetary dynamo to be driven by the $\alpha$-effect of inertial wave packets triggered by buoyant anomalies near the equatorial plane. The helical wave packets interact strongly with the cylindrical radial and azimuthal components of the field, lifting and twisting the transverse field lines. This $\alpha$-effect generates an emf which is parallel (anti-parallel) to the transverse field when the helicity is negative (positive) (Figure 1). Thus, the interaction of the wave packets with $B_{r}$ produces a quadrapolar poloidal current, which reinforces an anti-symmetric $B_{\theta}$. The waves interact with $B_{\theta}$ to produce a symmetric mean $J_{\theta}$, which through Ampère's law reinforces the poloidal component of the large-scale field. This completes the $\alpha^{2}$ dynamo cycle shown in Figure 1.

The generation of inertial wave packets by buoyant anomalies in the absence of a magnetic field is discussed in several papers (Davidson 2014; Davidson \& Ranjan 2015). Here, we study the dynamic effect of a uniform field on the properties of fast time-scale helical waves - paying particular attention to the kinetic, magnetic and cross helicities, and on the crucial emf and associated $\alpha$-effect. First, we map out the various kinds of waves that are supported by rapid rotation and a mean magnetic field.

\section{CHARACTERISTICS OF MAGNETIC-CORIOLIS WAVES}

Consider a rapidly rotating Boussinesq fluid into which we place a single buoyant blob with an initially Gaussian profile $\rho^{\prime} \sim \exp \left[-|\boldsymbol{x}|^{2} / \delta^{2}\right]$, which may evolve slowly under the influence of a uniform gravitational field. The background rotation of the fluid $\Omega=\Omega \boldsymbol{e}_{z}$ is orthogonal to gravity $\mathrm{g}=\mathrm{g} \boldsymbol{e}_{y}$, and we choose a reference frame which rotates with the fluid. Firstly, we review the purely hydrodynamic case studied in Davidson (2014). The buoyant blob spontaneously generates four columnar, helical vortices aligned with the rotation axis, which reach far above/below the initial disturbance (Figure 2). These vortices are in fact inertial wave packets with the dispersion relation $\varpi= \pm 2(\boldsymbol{k} \cdot \Omega) / k$ and group velocity $\boldsymbol{c}_{g}= \pm 2[\boldsymbol{k} \times(\boldsymbol{\Omega} \times \boldsymbol{k})] / k^{3}$ (note that $\boldsymbol{c}_{g}$ is the energy propagation velocity). Here $\boldsymbol{k}$ is the wave vector, $k=|\boldsymbol{k}|$, and the positive (negative) sign 
(a)

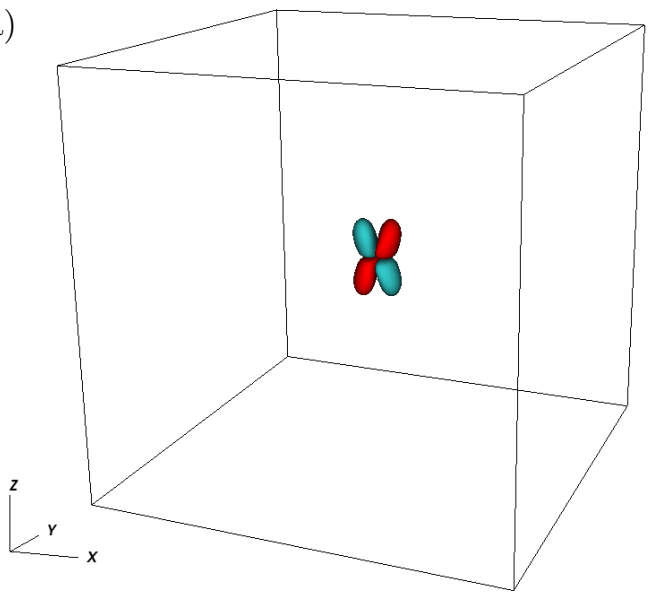

(c)

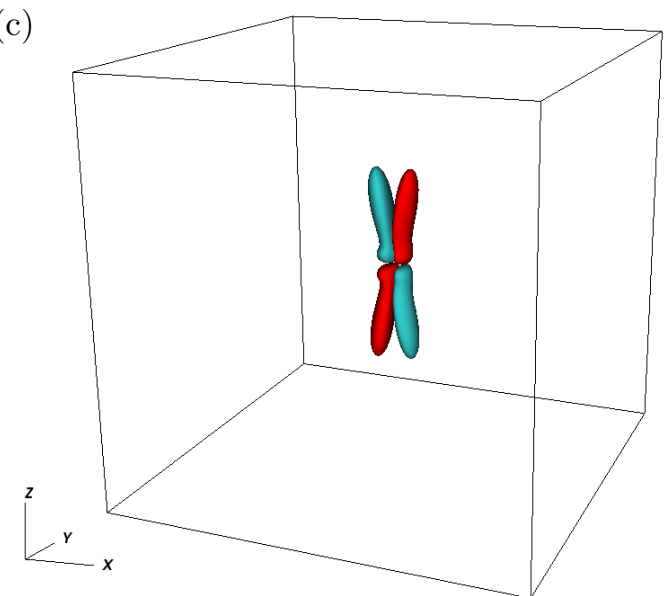

(b)

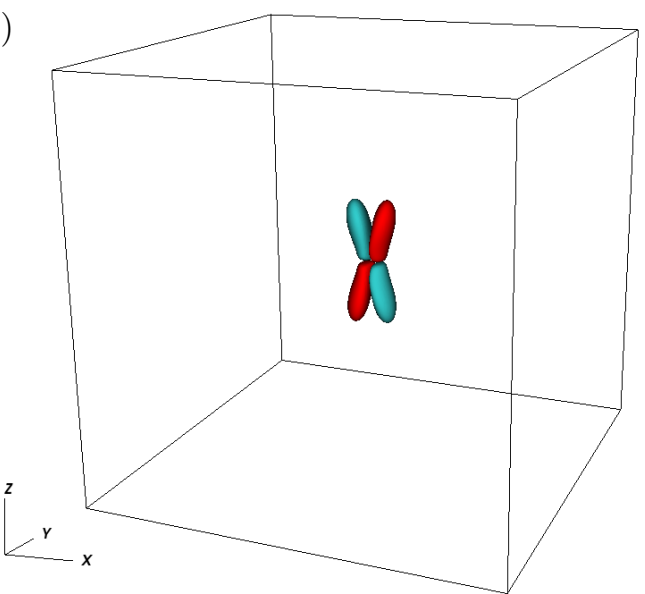

(d)

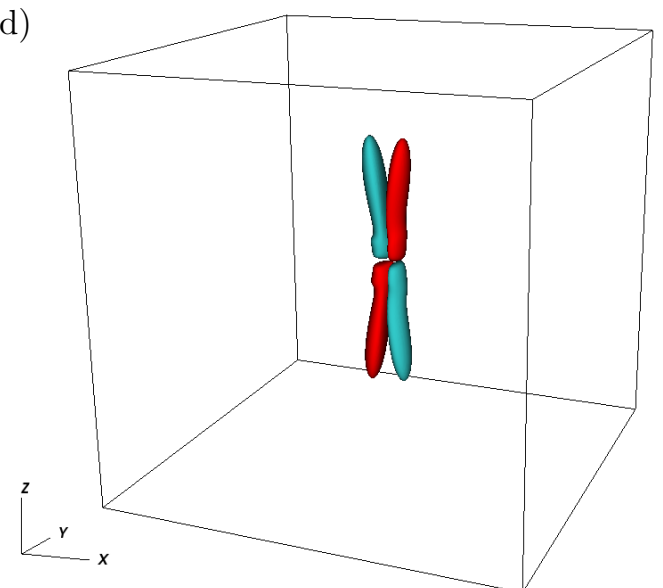

Figure 2. Inertial wave packets emitted from a buoyant anomaly in a rapidly rotating fluid: isosurfaces of axial velocity $u_{z}$ are shown at $\Omega t=$ (a) 2 , (b) 4 , (c) 6 , (d) 8 where red (blue) denotes positive (negative) $u_{z}$. The rotation axis is aligned with $z$. Four vortices are generated: two above the buoyant anomaly and two below. At $\Omega t=8$ the vortices are columnar in structure.

corresponds to upward (downward) travelling waves carrying negative (positive) kinetic helicity (Figure 3). For some blob size $\delta$, waves with a dominant wave number $k \sim \pi / \delta$ are typically dispatched. The segregation of helicity — negative (positive) above (below) the disturbance — is verified by direct numerical simulations (Davidson \& Ranjan 2015). A kinetic helicity distribution that is negative (positive) in the 'north' ('south') is reminiscent of geodynamo simulations (Schaeffer et al. 2017).

Why should the radiation pattern be so strongly concentrated above/below the disturbance, when the buoyant blob is initially spherical with no preferred direction? This question is explored in Davidson et al. (2006), and the crux of the explanation lies in the fact that $c_{g}$ is always per- 
(a)

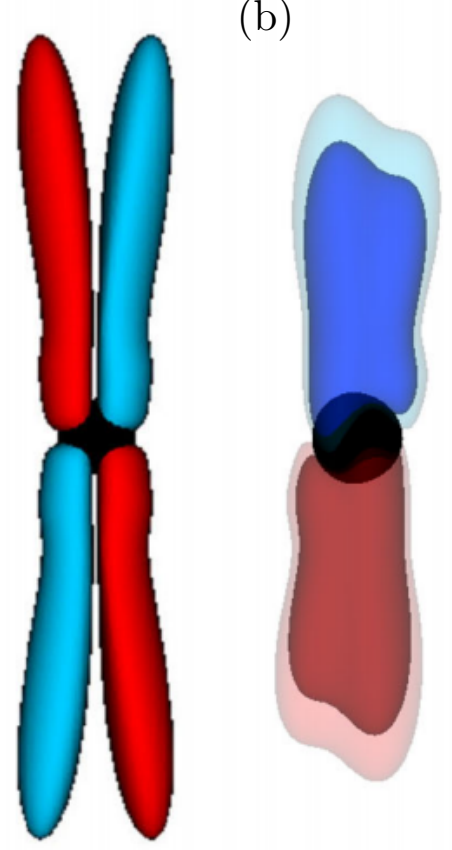

Figure 3. Inertial wave packets emitted from a buoyant anomaly (shown in black) in a rapidly rotating fluid. (a) Four vortices shown by isosurfaces of $u_{z}$ and (b) kinetic helicity isosurfaces; note the segregation of kinetic helicity negative (positive) above (below) the buoyant blob.

pendicular to $\boldsymbol{k}$. Kinetic energy transported by an inertial wave packet to some off-axis location is associated with only one wave vector within the source spectrum, and is propagated at a speed less than $2 \Omega / k$. However energy is radiated along the rotation axis at the highest possible speed $2 \Omega / k$, and is associated with a two-dimensional disc of wave vectors in the horizontal plane. The 'folding-up' of the two-dimensional disc (in $\boldsymbol{k}$-space) onto a one-dimensional thin cylinder (in real space) constitutes the robust channelling of energy along the rotation axis (Davidson 2013, §3.3.2). (For an alternative argument, based on angular momentum considerations, see Davidson et al. 2006).

In a planetary core, however, there often lies a dynamic magnetic field. The introduction of a mean magnetic field $\boldsymbol{B}_{0}$ modifies inertial waves into what we term magnetic-Coriolis (MC) waves, which encompasses a large variety of wave dynamics. Alfvén waves are now permitted to oscillate along magnetic field lines, with a group velocity $\boldsymbol{c}_{g}= \pm \boldsymbol{B}_{0}$ (we scale the magnetic field throughout by $\sqrt{\mu \rho}$ so that it is in velocity units). The combined effects of rotation and a 
background magnetic field may be educed through considering the example outlined above, with the addition of a mean field $\boldsymbol{B}_{0}=B_{0} \boldsymbol{e}_{x}$ orthogonal to both the rotation vector and gravity. The uniform field may be thought of as a local approximation to the large-scale East-West field in a planetary core. We first consider plane-wave solutions to the linearised diffusion-less equations (considered in detail in Bardsley \& Davidson 2016) to get an idea of what we might expect to observe in the simulations presented in $\S 3$ and $\S 4$. The linearised diffusion-less equations removed from the disturbance are

$$
\begin{gathered}
\partial_{t} \boldsymbol{b}=\left(\boldsymbol{B}_{0} \cdot \boldsymbol{\nabla}\right) \boldsymbol{u}, \quad \partial_{t} \boldsymbol{\omega}=\left(\boldsymbol{B}_{0} \cdot \boldsymbol{\nabla}\right) \boldsymbol{j}+2(\boldsymbol{\Omega} \cdot \boldsymbol{\nabla}) \boldsymbol{u}, \\
\boldsymbol{\omega}=\boldsymbol{\nabla} \times \boldsymbol{u}, \quad \boldsymbol{j}=\boldsymbol{\nabla} \times \boldsymbol{b},
\end{gathered}
$$

where $\boldsymbol{u}$ and $\boldsymbol{b}$ are the fluctuating velocity and magnetic fields, and the corresponding vorticity and current density fields are $\boldsymbol{\omega}$ and $\boldsymbol{j}$. We also introduce a solenoidal vector potential through $\boldsymbol{\nabla} \times \boldsymbol{a}=\boldsymbol{b}$. These equations combine to give the wave-like equation (Davidson 2005, pp. 531)

$$
\left[\frac{\partial^{2}}{\partial t^{2}}-\left(\boldsymbol{B}_{0} \cdot \boldsymbol{\nabla}\right)^{2}\right]^{2} \boldsymbol{\nabla}^{2} \boldsymbol{u}+4(\boldsymbol{\Omega} \cdot \boldsymbol{\nabla})^{2} \frac{\partial^{2} \boldsymbol{u}}{\partial t^{2}}=\mathbf{0} .
$$

If we apply the plane-wave ansatz $\boldsymbol{f}=\hat{\boldsymbol{f}} \exp [\mathrm{i}(\boldsymbol{k} \cdot \boldsymbol{x}-\varpi t)]$ (where $\boldsymbol{f} \sim \boldsymbol{u}, \boldsymbol{\omega}, \boldsymbol{a}, \boldsymbol{b}, \boldsymbol{j}$ ) to (3) we arrive at these relations for monochromatic plane-wave solutions to (3)

$$
\hat{\boldsymbol{\omega}}=\mp k \hat{\boldsymbol{u}}, \quad \hat{\boldsymbol{j}}=\mp k \hat{\boldsymbol{b}}, \quad \hat{\boldsymbol{b}}=-\frac{\varpi_{B}}{\varpi} \hat{\boldsymbol{u}}, \quad \hat{\boldsymbol{b}}=\mp k \hat{\boldsymbol{a}}
$$

and the dispersion relationship becomes

$$
\varpi^{2} \mp \varpi_{I} \varpi-\varpi_{B}^{2}=0
$$

where $\varpi_{B}=\boldsymbol{k} \cdot \boldsymbol{B}_{0}$ and $\varpi_{I}=2(\boldsymbol{k} \cdot \Omega) / k$ are the the Alfvén and inertial wave frequencies (Davidson 2013, pp. 150). It is clear from (4) that all plane-wave solutions to (3) have maximal kinetic, magnetic and cross helicity $-h_{k}=\boldsymbol{u} \cdot \boldsymbol{\omega}, h_{m}=\boldsymbol{a} \cdot \boldsymbol{b}$ and $h_{c}=\boldsymbol{u} \cdot \boldsymbol{b}$ respectively. This means that (for monochromatic plane-wave solutions) the pairs of fields: $(\boldsymbol{u}, \boldsymbol{\omega}),(\boldsymbol{b}, \boldsymbol{a})$ and $(\boldsymbol{u}, \boldsymbol{b})$ are exactly aligned, though this need not be true for a wave packet (Davidson \& Ranjan 2015). Further, as $h_{k}$ is maximal irrespective of the presence of a mean field, this tells us that all monochromatic MC waves have an identical structure for the velocity field - a circularly polarised transverse wave (Moffatt 1978). 
The group velocity for these waves, acquired from (5), can be expressed in the form

$$
\left[1+\left(\frac{\varpi_{B}}{\varpi}\right)^{2}\right] \boldsymbol{c}_{g}=\frac{2 \varpi_{B}}{\varpi} \boldsymbol{B}_{0}+\boldsymbol{c}_{g_{I}},
$$

where $c_{g_{I}}= \pm 2[\boldsymbol{k} \times(\boldsymbol{\Omega} \times \boldsymbol{k})] / k^{3}$. From (6), we see that energy with $\boldsymbol{k}$ perpendicular to $\Omega$ is still focused onto the rotation axis (as it is for pure inertial waves), however we now have a component of the group velocity in the direction of the mean field.

In the rapidly rotating regime of Earth's outer core, the conventional wisdom states that on large scales we might expect $\varpi_{I} \gg \varpi_{B}$. This produces a splitting of the wave frequencies: $\varpi \approx\left|\varpi_{I}\right|$ and $\varpi \approx \varpi_{B}^{2} /\left|\varpi_{I}\right|$. The former corresponds to weakly modified off-axis inertial waves, which might traverse the core on a timescale of weeks, and the latter to magnetostrophic waves, perturbations which predominantly migrate along the mean-field on a timescale of centuries (Moffatt 1978) (see the end members in Figure 4). Due to their low frequencies and slow group velocities, magnetostrophic waves have been covered at length in the literature in efforts to explain the secular variation of the geomagnetic field (Hide 1966; Malkus 1967). In the general case, exactly how the energy is dispersed depends strongly on the orientation of $\boldsymbol{\Omega}, \boldsymbol{k}$, and $\boldsymbol{B}_{0}$. However, as it became clear for pure inertial waves, we might expect that there is special role for those waves with $k$ perpendicular to $\Omega$; indeed this is a degenerate case for the magnetostrophic wave analysis above (Bardsley \& Davidson 2016).

From (5), the frequencies of the waves with $\boldsymbol{k} \cdot \Omega \approx 0$ match the Alfvén frequency: $\varpi \approx \pm \varpi_{B}$. The leading order expression for the group velocity, for upward propagating waves, becomes

$$
\boldsymbol{c}_{g}= \pm \boldsymbol{B}_{0}+\frac{\Omega}{k}
$$

An Alfvén-like propagation occurs along large-scale field lines, and the wave-energy is propagated axially at half the speed of low-frequency inertial waves. These waves are termed Inertial-Alfvén (IA) waves, and were first highlighted by Bardsley \& Davidson (2016) as a possible means of establishing quasi-geostrophy in planetary cores. The self-focusing property of pure inertial waves is passed over to IA waves in the presence of a transverse uniform field, as all waves with $\boldsymbol{k}$. $\Omega \approx 0$, which may be initially launched as inertial waves, are converted to IA waves as soon as they feel the effects of the field. Any weakly modified inertial waves emitted must be off- 


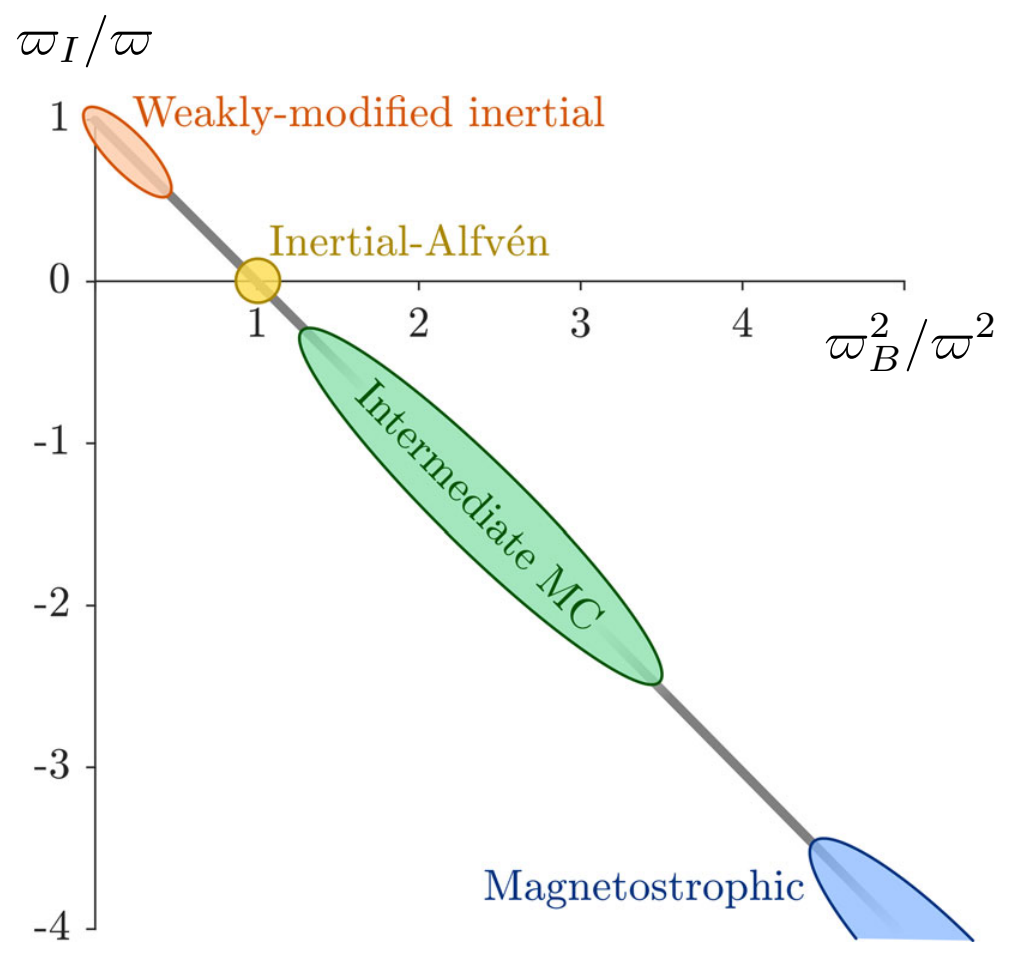

Figure 4. The classes of waves derived from the MC wave dispersion relation (5); after Bardsley \& Davidson (2017).

axis waves, which have a lower energy density. From (4), all MC waves have maximal $h_{k}, h_{m}$ and $h_{c}$, however IA waves propagate energy in equipartition i.e. $\boldsymbol{u}^{2}=\boldsymbol{b}^{2}$. This is in contrast to weakly modified inertial waves that are characterised by maximal $h_{k}$, but $h_{m} \approx 0, h_{c} \approx 0$ and $\boldsymbol{b}^{2} / \boldsymbol{u}^{2} \approx 0$, and slow magnetostrophic waves with maximal $h_{m}$, but $h_{k} \approx 0, h_{c} \approx 0$ and $\boldsymbol{u}^{2} / \boldsymbol{b}^{2} \approx 0$. The wave types derived from the dispersion relation (5) are summarised in Figure 4. Here, the 'intermediate MC' region characterises those wave packets which sit between the selffocusing IA waves and slow magnetostrophic waves. The dispersion properties of intermediate MC waves depend strongly on the ratio of the Alfvén and inertial frequencies - at Earth-like values of this ratio the intermediate waves predominantly radiate information along the direction of the rotation axis (Bardsley \& Davidson 2017).

One final point arises from taking the dot product of (6) with $\Omega$. Using (4), it can be shown that the segregation of $h_{k}$ for pure inertial waves - negative (positive) above (below) the disturbance — extends to MC waves, only now $h_{m}$ is segregated in exactly the same way. Evidently this is true for modified inertial waves, magnetostrophic waves and IA waves alike. Cross helicity is 
maximal for IA waves, however there is no such segregation of $h_{c}$ above/below the source, there is a left-right asymmetry which may be seen from (4) and the fact that the sign of $\varpi_{B}$ depends on $\boldsymbol{B}_{0} \cdot \boldsymbol{k}$.

\section{DISPERSION OF WAVES FROM A SINGLE BUOYANT BLOB}

Before looking at the dispersion of waves from a random sea of buoyant anomalies $(\S 4)$ it is instructive to consider the case of a single buoyant blob. Our local numerical set-up is reminiscent of the equatorial regions of a planetary outer core, where gravity points radially inward and the rotation vector is vertical (similar to Davidson \& Ranjan 2015; McDermott \& Davidson 2019). We focus our investigations on a local approximation of a toroidal field, perpendicular to both gravity and rotation. We introduce three simulations in a Cartesian domain of an electrically conducting Boussinesq fluid with an applied uniform magnetic field $B_{0} \boldsymbol{e}_{x}$, background rotation $\Omega \boldsymbol{e}_{z}$, and constant gravity g $\boldsymbol{e}_{y}$. The equations are

$$
\begin{gathered}
\partial_{t} \boldsymbol{u}+(\boldsymbol{u} \cdot \boldsymbol{\nabla}) \boldsymbol{u}+2 \Omega\left(\boldsymbol{e}_{z} \times \boldsymbol{u}\right)=-\boldsymbol{\nabla} \Pi+\mathrm{g} c \boldsymbol{e}_{y}+B_{0} \partial_{x} \boldsymbol{b}+(\boldsymbol{b} \cdot \boldsymbol{\nabla}) \boldsymbol{b}+\nu \boldsymbol{\nabla}^{2} \boldsymbol{u}, \\
\partial_{t} \boldsymbol{b}+(\boldsymbol{u} \cdot \boldsymbol{\nabla}) \boldsymbol{b}=B_{0} \partial_{x} \boldsymbol{u}+(\boldsymbol{b} \cdot \boldsymbol{\nabla}) \boldsymbol{u}+\eta \boldsymbol{\nabla}^{2} \boldsymbol{b}, \\
\partial_{t} c+(\boldsymbol{u} \cdot \boldsymbol{\nabla}) c=\kappa \nabla^{2} c, \\
\boldsymbol{b}=\boldsymbol{\nabla} \times \boldsymbol{a}, \boldsymbol{\nabla} \cdot \boldsymbol{u}=\boldsymbol{\nabla} \cdot \boldsymbol{b}=\boldsymbol{\nabla} \cdot \boldsymbol{a}=0,
\end{gathered}
$$

where the reduced pressure $\Pi$ takes into account magnetic pressures and centrifugal accelerations and $c=\rho^{\prime} / \rho$ is the dimensionless density perturbation. We remind the reader that the magnetic field is scaled by $\sqrt{\mu \rho}$, so that it is in velocity units. The magnetic diffusivity $\eta$, kinematic viscosity $\nu$, and the scalar diffusivity $\kappa$ of the fluid are all constant and equal, thus the thermal and magnetic Prantdl numbers are $\operatorname{Pr}=P m=1$. The equations are solved using standard pseudo-spectral techniques, with a 2/3 de-aliasing truncation and a second-order Runge-Kutta time advance scheme (Gómez et al. 2005). The triply periodic simulation box is extended in the direction of the rotation axis, to allow wave packets to propagate for up to 40 rotation periods before reaching the top/bottom of the box.

The initial conditions are $\boldsymbol{u}=\boldsymbol{b}=\mathbf{0}$, with a single spherical density anomaly at the origin, 
which has an initially Gaussian profile $c=\rho^{\prime} / \rho \sim \exp \left[-|\boldsymbol{x}|^{2} / \delta^{2}\right]$, but evolves according to an advection-diffusion equation thereafter. Here $\boldsymbol{x}$ is the position vector and $\delta$ is the blob size. Compared to the blob size, the box spans $[-25 \delta, 25 \delta]$ in the horizontal directions and $[-75 \delta, 75 \delta]$ in the direction of the rotation vector. The ratio of Alfvén to inertial frequencies is expressed by the Lehnert number $L e=B_{0} / \Omega \delta$, and the Lundquist number $L u=B_{0} \delta / \eta$ measures the ratio of the magnetic diffusion time-scale to the time-scale of Alfvén waves, where $\eta$ is the magnetic diffusivity. Estimates of $L e$ and $L u$ for the small-scales in Earth's outer core lie in the ranges $L e \sim 0.001-0.1$ and $L u \sim 100-1000$. These estimates are very sensitive to the hidden azimuthal magnetic field strength ( $\sim 3 \mathrm{mT}$, Gillet et al. 2010), and the flow length-scale perpendicular to the rotation axis ( $\sim 10 \mathrm{~km}$, Davidson 2014). Note that the convective length scale in Earth's core was recently estimated at $\sim 30 \mathrm{~km}$ (Guervilly et al. 2019), which results in $L e \sim 0.01$. A parameter often referenced in the literature, which does not include a length scale, is the Elsasser number $\Lambda=L e L u$, and we expect $\Lambda \sim 10$ for Earth's dynamo. First, we set $L e=0.1$ and $L u=160$ (so $\Lambda=16$ ), as these choices illustrate the key wave dynamics in a reasonable amount of simulation time. (See also simulation S4 with the same parameters later in $\S 4$ ).

Wave packets radiate away from the buoyant blob, and extend in the direction of the rotation vector, as in the hydrodynamic case (Figure 2). We stop the simulation after $30 \Omega t$ and inspect the dispersion pattern, as illustrated in Figure 5. We show isosurfaces of $\pm u_{z}$ coloured by relative kinetic helicity $h_{k}^{*}=h_{k} /|\boldsymbol{u}||\boldsymbol{\omega}|$, relative magnetic helicity $h_{m}^{*}=h_{m} /|\boldsymbol{a}||\boldsymbol{b}|$, relative cross helicity $h_{c}^{*}=h_{c} /|\boldsymbol{u}||\boldsymbol{b}|$ and the normalised emf in the $x$-direction: $\mathcal{E}_{x}^{*}=(\boldsymbol{u} \times \boldsymbol{b})_{x} /|\boldsymbol{u} \times \boldsymbol{b}|$. From (7), we expect to see axially elongated wave packets with a high energy density, that have moved horizontally to $\pm x / \delta \approx L e(\Omega t) \approx 3$. The isosurfaces shown in Figure 5 are consistent with this prediction. As expected from the earlier analysis, kinetic and magnetic helicity are cleanly segregated negative (positive) above (below) the buoyant blob. The normalised fluctuating emf in the $x$-direction, $\mathcal{E}_{x}^{*}$, is segregated in the opposite way to $h_{k}$ and $h_{m}$, with positive (negative) emf being transported above (below) the blob. The normalised emf has a smaller magnitude than the relative helicities, and $h_{k}^{*}$ and $h_{m}^{*}$ are larger in magnitude than $h_{c}^{*}$. The buoyancy field itself does 

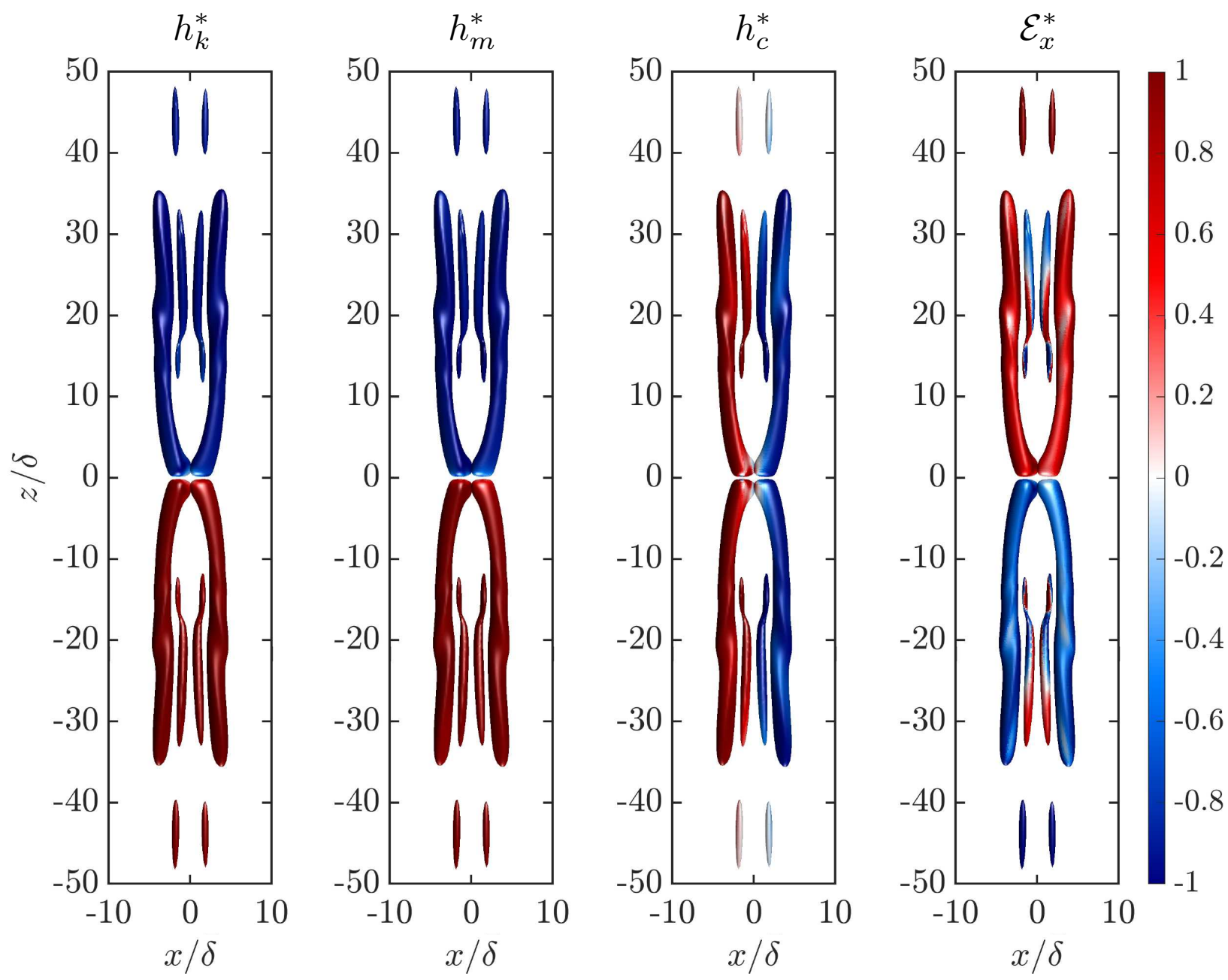

Figure 5. Buoyant blob source at $L e=0.1$. Axial velocity isosurfaces at $\Omega t=30$ coloured by relative kinetic helicity $h_{k}^{*}$, relative magnetic helicity $h_{m}^{*}$, relative cross helicity $h_{c}^{*}$ and the normalised emf in the $x$-direction $\mathcal{E}_{x}^{*}$ (left to right).

not appreciably evolve, owing to the low value of the Rossby number $R o=u / 2 \Omega \delta \approx 0.01$, where $u$ is the characteristic velocity magnitude.

The cross helicity and the emf are the dot product and cross product of $\boldsymbol{u}$ and $\boldsymbol{b}$. It follows that their values are closely related through $\left|h_{c}\right|^{2}+|\mathcal{E}|^{2}=|\boldsymbol{u}|^{2}|\boldsymbol{b}|^{2}$. As the quantities are squared in this relation, even when the relative cross helicity holds a large value, say $\approx 0.75$, the normalised emf would be respectively $\approx 0.66$. With the mean-field in the $x$-direction we expect $\mathcal{E}_{x}$ to dominate the other components of the emf, and this is what we observe for the current example (Figure 5). The wave packets generated by a single blob are permitted to induce a strong emf, in spite of the relatively large degree of alignment between $\boldsymbol{u}$ and $\boldsymbol{b}$. 

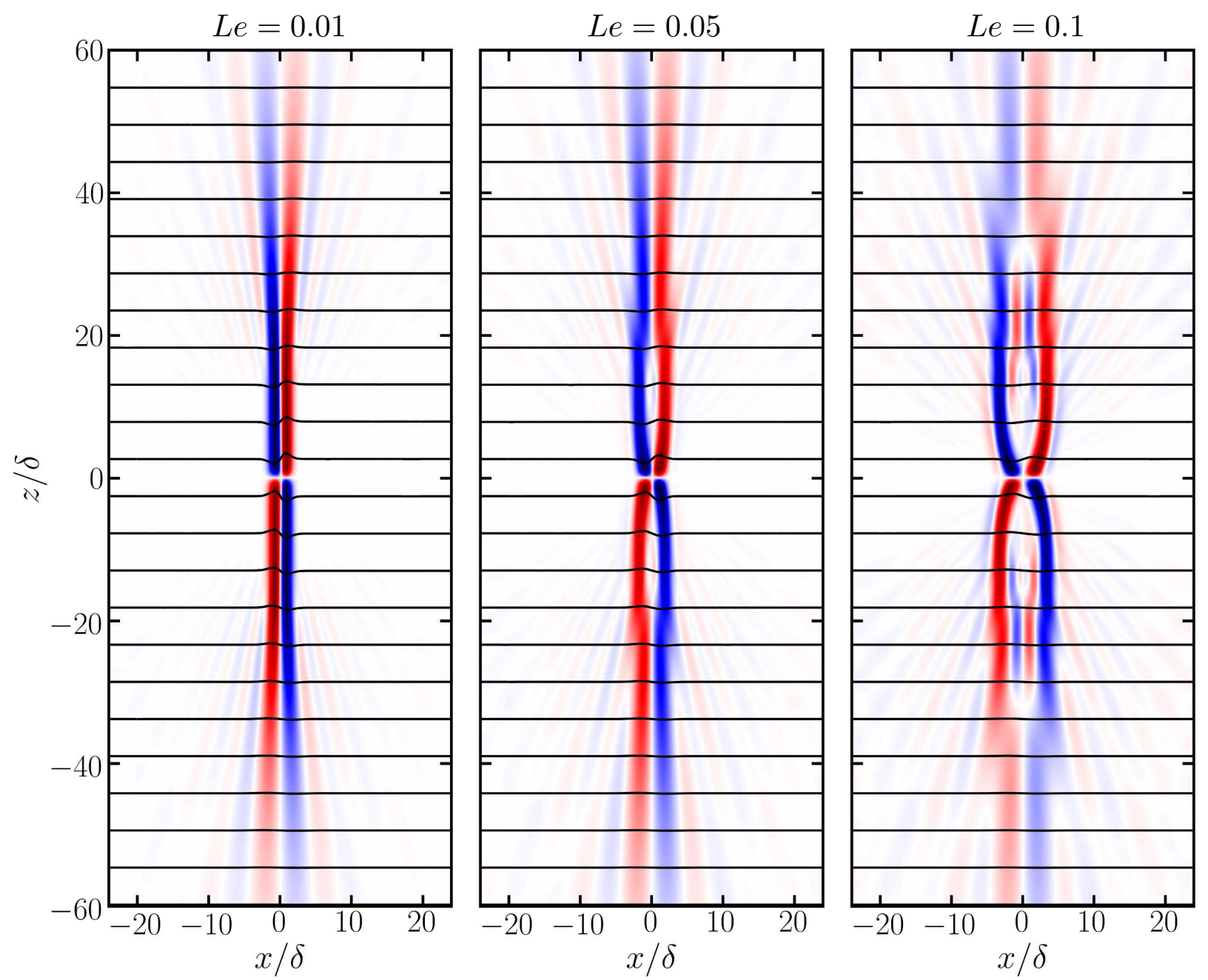

Figure 6. $u_{z}$ in the plane $y=0$ at $\Omega t=30$, gravity points into the page and the colour scale is saturated at $\pm 85 \%$ of the maximum of $\left|u_{z}\right|$ in each pane. Blue (red) is negative (positive) $u_{z}$, the horizontal black lines are $\boldsymbol{B}_{0}+\boldsymbol{b}$ and the Lehnert number increases from left to right. The perturbations to the field lines have been exaggerated by a factor of 10 .

Now we explore the effect of varying the Lehnert number, or equivalently the mean field strength. In Figure 6 we show the axial velocity at $\Omega t=30$ with the field lines $\boldsymbol{B}_{0}+\boldsymbol{b}$ superimposed, in the plane $y=0$ for three Lehnert numbers $L e=0.01,0.05,0.1$. This choice of parameters is equivalent to the simulations initialised with a layer of buoyancy: S2, S3 and S4 introduced later in $\S 4$. For $L e=0.01$ (left), the magnetic field barely effects the wave dispersion, so we see transient Taylor columns growing along $\boldsymbol{e}_{z}$, similar to those seen in purely hydrodynamic simulations (Davidson \& Ranjan 2015; McDermott \& Davidson 2019). Weaker petals of off-axis energy are also present, due to the isotropic source spectrum, however the energy density is highest 
on-axis. With a stronger uniform field (say $L e=0.1$ ), there is evidently a considerable effect of the magnetic field on the wave dispersion. We see similar columnar structures, but now the energy is displaced laterally off-axis due to the component of $\boldsymbol{c}_{g}$ parallel to $\boldsymbol{B}_{0}$, see (7). Note that the dispersion pattern observed here is indistinguishable from the diffusionless analytical results of Bardsley \& Davidson (2016), with a static buoyant blob at $L e=0.1$. It will be important to note that for the single blob case, the wave packets propagate horizontally away from each other, and do not interact (Figure 6). The horizontal propagation should not be mistaken for the slow evolution of magnetostrophic waves, whose time-scale is vastly greater than $30 \Omega t$ - the dynamics shown occur on the fast time-scale.

A planetary core will inevitably be packed with buoyant anomalies, slowly migrating from the centre outwards under the influence of gravity. This is not dependent on whether the convection is driven thermally or compositionally. Each one of these buoyant blobs is obliged to emit a spectrum MC waves, carrying varying degrees of helicity (kinetic, magnetic or cross). Similarly to the hydrodynamic case, it is the self-focusing waves launched with $k \cdot \Omega \approx 0$ which are particularly potent at creating columnar structures (Figures $5 \& 6$ ), and in the presence of a uniform field it is the inertial and IA waves that assume this role. It is observed that in many of the published dynamo simulations, the root-mean-square (r.m.s.) radial velocity (Sakuraba \& Roberts 2009) and the temperature/density perturbations (Olson et al. 1999; Schaeffer et al. 2017) are concentrated near the equatorial plane $(\S 1)$. This observation motivates the initial conditions for the simulations presented in the remainder of the paper.

\section{NUMERICAL SIMULATIONS WITH A LAYER OF BUOYANT ANOMALIES}

We present six simulations with the same set-up as in $\S 3$, however the initial condition for the buoyancy field in this section is now a layer of buoyant anomalies rather than a single buoyant blob. The simulations are initialised with $\boldsymbol{u}=\boldsymbol{b}=\mathbf{0}$, and with a localised layer of buoyant anomalies in the vertical centre of the box. Each constituent buoyant blob is similar to the single buoyant source in the previous section, with $c_{i} \sim \exp \left[-\left(\boldsymbol{x}-\boldsymbol{x}_{i}\right)^{2} / \delta^{2}\right]$, where $\boldsymbol{x}_{i}$ locates the blob centre and $\delta$ is the blob size. The blob sizes are chosen from a uniform distribution about 
Table 1. Simulation parameters and estimated local values for Earth's outer core.

\begin{tabular}{|c|c|c|c|c|c|}
\hline & Run & $L e$ & $L u$ & Ro & $\Lambda=L e L u$ \\
\hline Not Earth-like $L e$ & S1 & $10^{-8}$ & $1.6 \times 10^{-5}$ & $2.8 \times 10^{-3}$ & $10^{-13}$ \\
\hline \multirow[t]{2}{*}{ Earth-like $L e$} & S2 & 0.01 & 16 & $2.7 \times 10^{-3}$ & 0.16 \\
\hline & S3 & 0.05 & 78 & $1.9 \times 10^{-3}$ & 3.9 \\
\hline \multirow[t]{2}{*}{ Marginal Le } & S4 & 0.1 & 160 & $1.4 \times 10^{-3}$ & 16 \\
\hline & S5 & 0.2 & 310 & $1.0 \times 10^{-3}$ & 62 \\
\hline \multirow[t]{2}{*}{ Not Earth-like $L e$} & S6 & 0.5 & 780 & $7.9 \times 10^{-4}$ & 390 \\
\hline & Earth & $0.001-0.1$ & $100-1000$ & $\sim 1 \times 10^{-4}$ & $\sim 10$ \\
\hline
\end{tabular}

the mean blob size $\bar{\delta}$, such that $\bar{\delta} / 2<\delta<3 \bar{\delta} / 2$ creating a diverse random cloud of buoyancy. Here, the box spans $[-25 \bar{\delta}, 25 \bar{\delta}]$ in the horizontal directions and $[-75 \bar{\delta}, 75 \bar{\delta}]$ in the direction of the rotation vector. We restrict the blob centres $\boldsymbol{x}_{i}$ to the layer $-2 \bar{\delta}<z<2 \bar{\delta}$, and they fill the box in the plane normal to $\boldsymbol{e}_{z}$. There are 2000 blobs within the layer, resulting in a filling factor of $50 \%$. Using the r.m.s. velocity in the mid-plane of the box $u_{0}$ we define a Reynolds number $R e=u_{0} \bar{\delta} / \nu \sim 20-50$. As $P m=1$ the magnetic Reynolds number is $R m=R e$, while the Ekman number is $E k=\nu / 2 \Omega \bar{\delta}^{2} \sim 2 \times 10^{-4}$.

We vary the Lehnert number $L e$ from the basically hydrodynamic value of $10^{-8}$ through to $L e=0.5$ by increasing the strength of the applied field, with the exact values given in table 1. Also reported are estimated values for the small scales in Earth's outer core. We also list the Lundquist number, defined as $L u=B_{0} \bar{\delta} / \eta$, which is expected to be large in planetary cores. We introduce an 'average over the wave packets' which we will use several times the remainder of the paper, which is taken over the region $|\boldsymbol{u}|+|\boldsymbol{b}|>0.05 \times \max (|\boldsymbol{u}|+|\boldsymbol{b}|)$. Using this average we define a velocity scale $u$, and the corresponding values of $R_{o}=u / 2 \Omega \bar{\delta}$ for our simulations are given in table 1 . These decrease from S1-6 as a result of kinetic energy being converted to magnetic energy for larger applied field strengths, and enhanced Ohmic dissipation. We will first qualitatively describe the results of simulations S1-6, then examine features of them in detail.

Figures $7 \& 8$ show isosurfaces of the axial velocity at \pm 2 standard deviations, coloured by $h_{k}^{*},\left|h_{c}^{*}\right|$ and $\mathcal{E}_{x}^{*}$ at $\Omega t=30$. Column four in Figures $7 \& 8$ displays a $y$-average (denoted $\langle\sim\rangle_{y}$ ) 


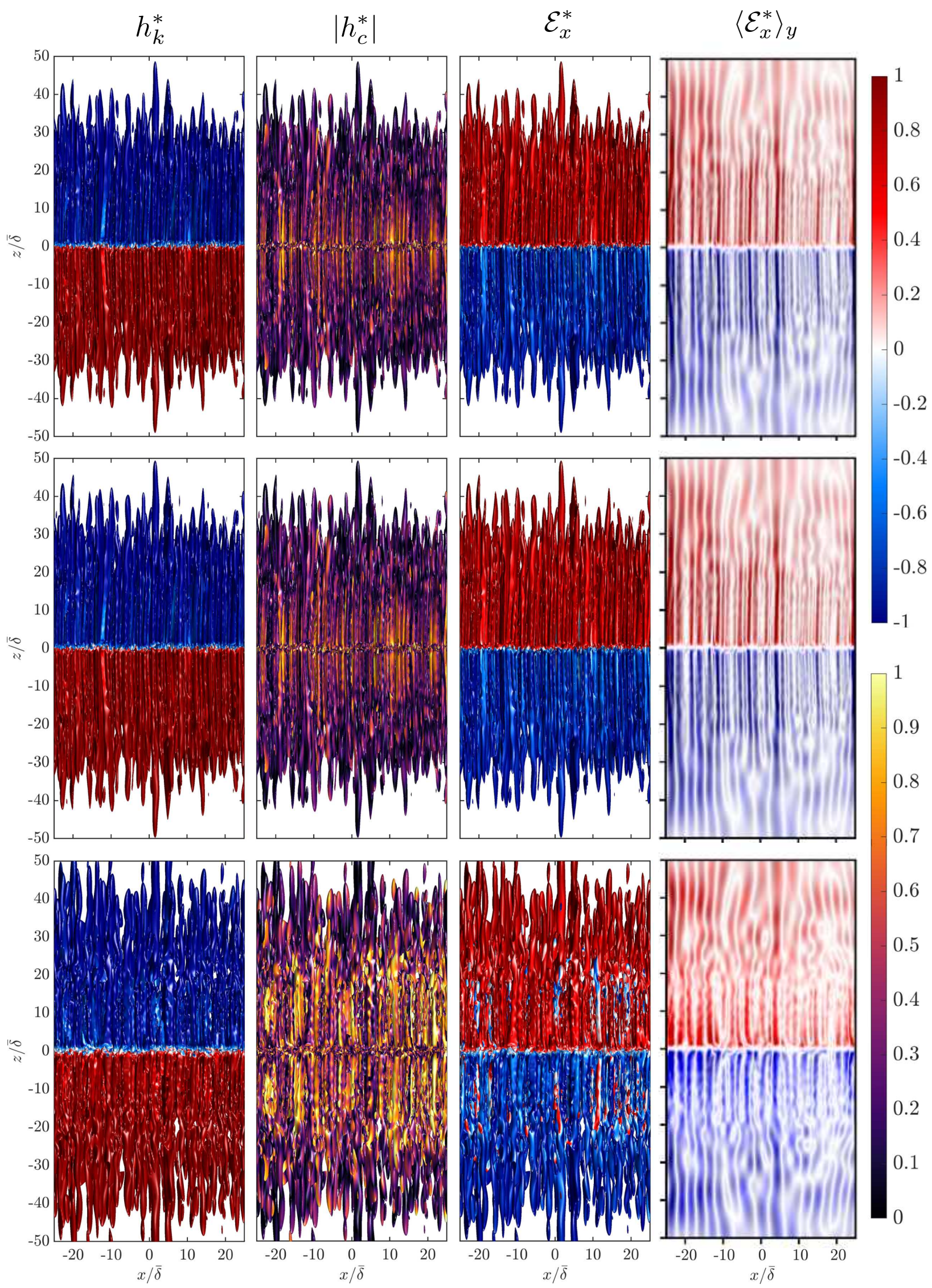

Figure 7. Axial velocity isosurfaces coloured by relative kinetic helicity $h_{k}^{*}$, relative cross helicity magnitude $\left|h_{c}^{*}\right|$ and normalised emf in the $x$-direction $\mathcal{E}_{x}^{*}$ (left to right). Isosurfaces shown for S1-3 (top to bottom), at \pm two standard deviations of $u_{z}$ and $\Omega t=30$. Column 4 shows a $y$-average where the colour intensity is controlled by $\left\langle\left|u_{z}\right|\right\rangle_{y}$ and the hue is set by $\left\langle\mathcal{E}_{x}^{*}\right\rangle_{y}$. Note the top/bottom of the box are at $\pm 75 z / \bar{\delta}$. 

$h_{k}^{*}$
$\left|h_{c}^{*}\right|$
$\mathcal{E}_{x}^{*}$
$\left\langle\mathcal{E}_{x}^{*}\right\rangle_{y}$
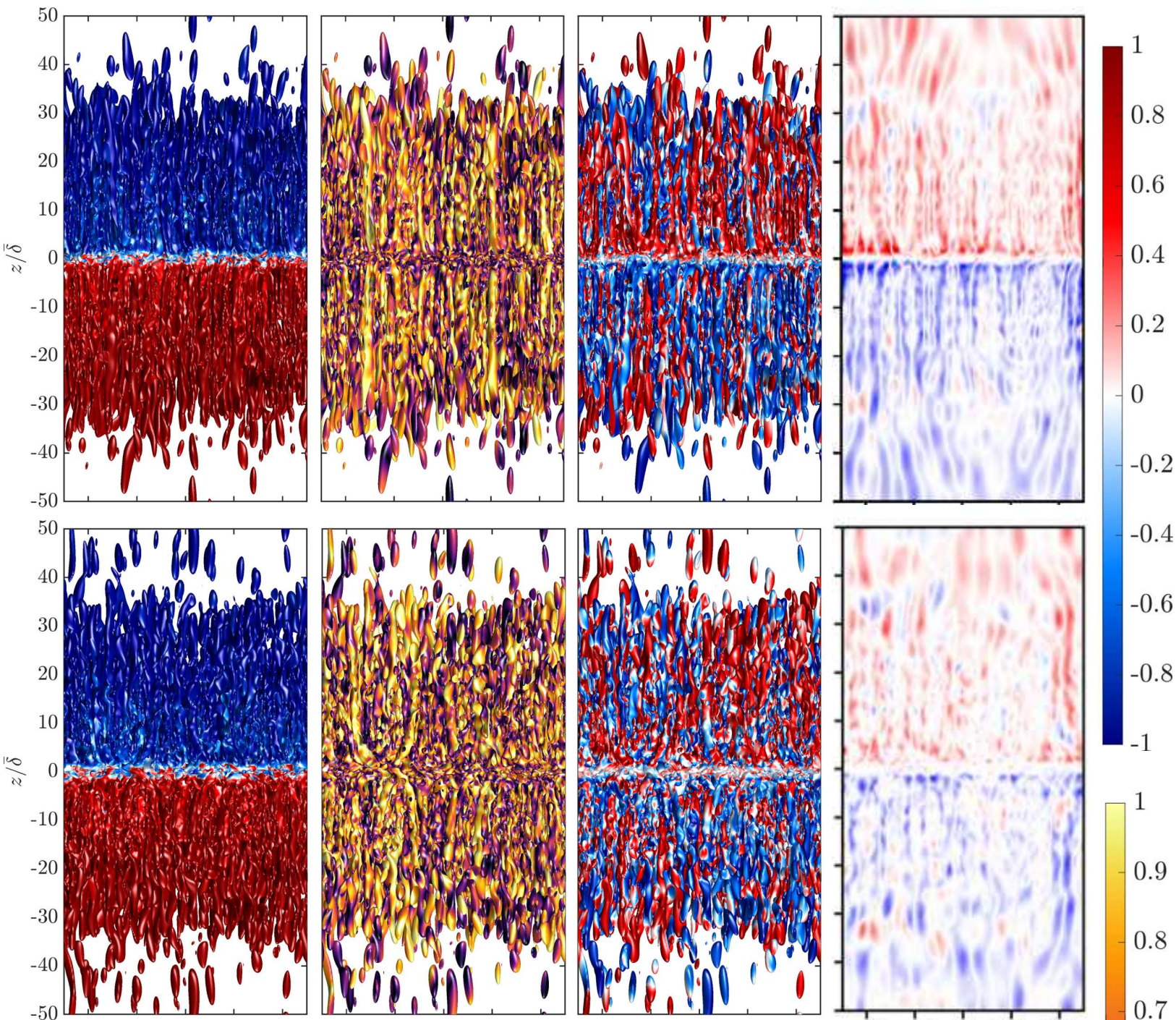

$-0.4$
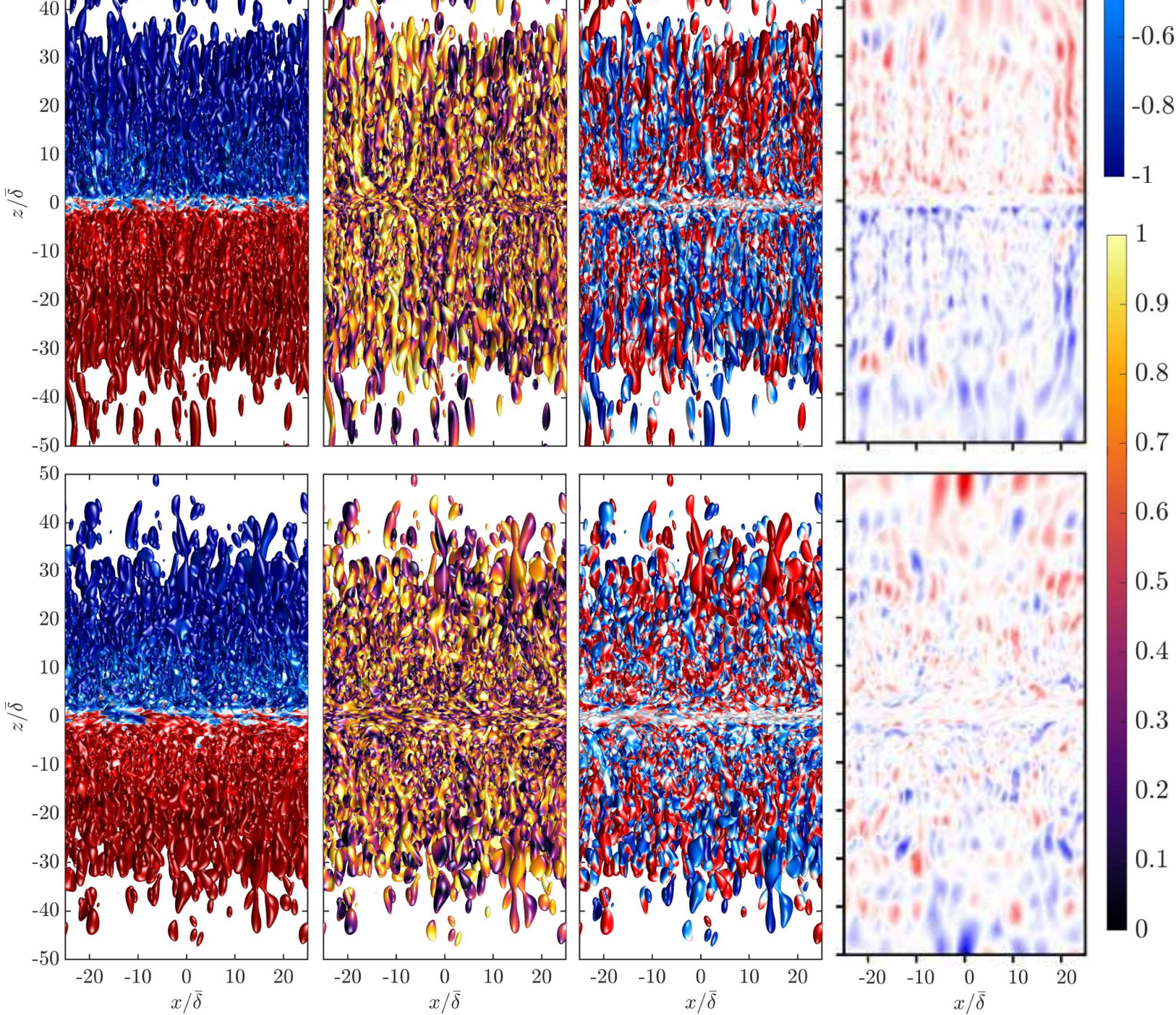

Figure 8. Same as Figure 7, for S4-6 (top to bottom). 


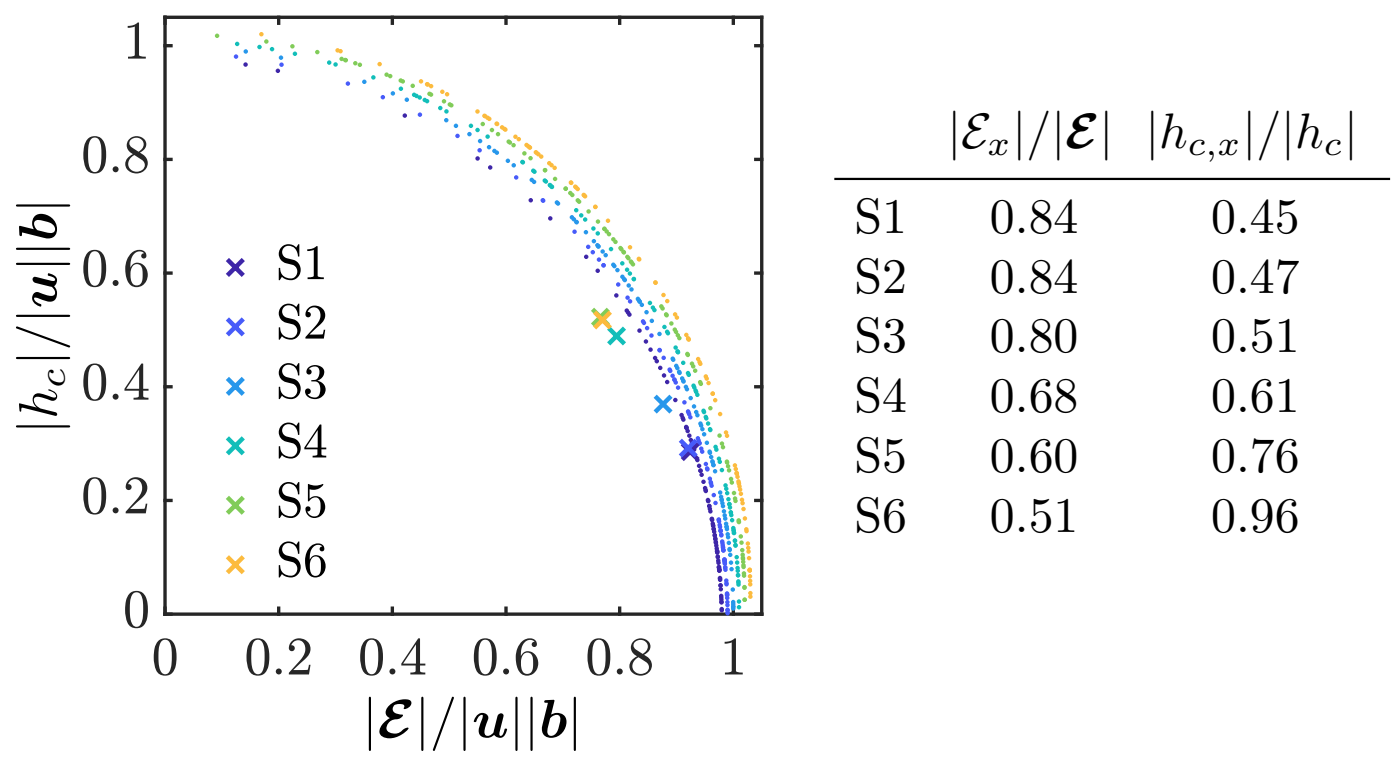

Figure 9. The relation between cross helicity and the induced emf for a random selection of points within the wave packets at $\Omega t=20$. All points for each simulation lie on the unit circle, but they have been shifted slightly for clarity. The crosses indicate the average over the wave packets, and the markers for S1 and S2 and for S5 and S6 are nearly on top of one another. The ratios $\left|\mathcal{E}_{x}\right| /|\mathcal{E}|$ and $\left|h_{c, x}\right| /\left|h_{c}\right|$ averaged over the wave packets at $\Omega t=20$ are listed to the right.

where the colour intensity is controlled by $\left\langle\left|u_{z}\right|\right\rangle_{y}$ and the hue is set by $\left\langle\mathcal{E}_{x}^{*}\right\rangle_{y}$, which illustrates how coherent the emf is throughout the wave packets. For $L e<0.1$ (S1-3), the buoyant layer in the vertical centre of the box emits a spectrum of wave packets which form columnar structures aligned with the rotation axis. These structures are very similar to those seen in Figures $5 \& 6$ for the single blob case. Those columnar structures in Figure 7 resemble weakly modified inertial waves for $\mathrm{S} 1$ and $\mathrm{S} 2$; in fact the images for $\mathrm{S} 1$ and $\mathrm{S} 2$ (at $L e=10^{-8}$ and $L e=0.01$ ) are almost identical. This is our first observation: that on timescales on the order of one week, MC waves in the regime $L e \lesssim 0.01$ are not strongly affected by the mean-field, and behave much the same as inertial waves. For S3 ( $L e=0.05)$, the wave-field remains largely coherent in the axial direction, as expected for a small value of $L e$. There is an increase in $\left|h_{c}^{*}\right|$ for wave packets with a slower axial group velocity, however the normalised emf remains strong and well segregated.

We tentatively identify inertial wave packets travelling at roughly the speed of low-frequency inertial waves, but in regions where we observe increased levels of cross helicity, IA wave packets must also be present. Intermediate MC waves must be present, however they are more difficult to 
classify as they are characterised by a lower energy density and varying degrees of cross helicity, though at low-Le these wave packets will be broadly columnar in structure by virtue of (6). Simulation S4 is in the same regime $(L e=0.1)$ as the individual blob example shown in Figure 5. A closer inspection of Figure 8 reveals that the wave packet morphology observed in Figure 5 is present in the buoyant layer case, where the wave packets elongate along the rotation axis meanwhile migrating along magnetic field lines, yet for multiple blobs the wave packets overlap in space. For $\mathrm{S} 4-6$ ( $L e \geq 0.1$ ), the picture exhibited for $\mathrm{S} 1-3$, columnar inertial/IA wave packets carrying intense positive (negative) $\mathcal{E}_{x}^{*}$ above (below) the buoyant layer becomes more complex, and there is notably more overlap between neighbouring wave packets. However, the $y$-average shown in column four of Figures $7 \& 8$ remains strongly positive (negative) above (below) the buoyant layer for $L e \lesssim 0.1$.

The wave packets become less coherent in the direction of the rotation axis as the uniform field strength is increased (Figure 8). For larger mean field strengths there is faster propagation in the direction of the imposed field. For S4, at $L e=0.1$, although there is enhanced cross helicity, the normalised emf appears to be mostly positive (negative) above (below) the source and of moderate magnitude; indeed the $y$-average is very coherent. This follows from the argument on pp. 12, which states that wave packets can induce a large emf in spite of carrying a lot of cross helicity. We show the relationship between $\left|h_{c}\right|$ and $|\mathcal{E}|$ for a random selection of points (plotted as dots) within the wave packets in Figure 9, the crosses indicate the average over the wave packets. The points for each simulation have been shifted slightly for clarity, but they all lie on the unit circle. The average normalised emf decreases with increasing $L e$ and the relative cross helicity increases. The same is true for the ratios $\left|\mathcal{E}_{x}\right| /|\mathcal{E}|$ and $\left|h_{c, x}\right| /\left|h_{c}\right|$ listed to the right in Figure $9, \mathcal{E}_{x}$ becomes less dominant and $h_{c, x}=u_{x} b_{x}$ becomes more dominant with increasing $L e$.

For $L e \geq 0.2$ (S5 and $\mathrm{S} 6$ ) the emf is weaker, less organised and appears to be uncorrelated with the kinetic helicity. The kinetic and magnetic helicities remain mostly segregated negative (positive) above (below) the buoyant layer for larger mean field strengths, but the segregation of the emf is no longer so clean, and this results in a weakening of $\left\langle\mathcal{E}_{x}^{*}\right\rangle_{y}$ (column four).

Davidson \& Ranjan (2015) confirmed that inertial wave packets launched from a layer of buoy- 
(a)

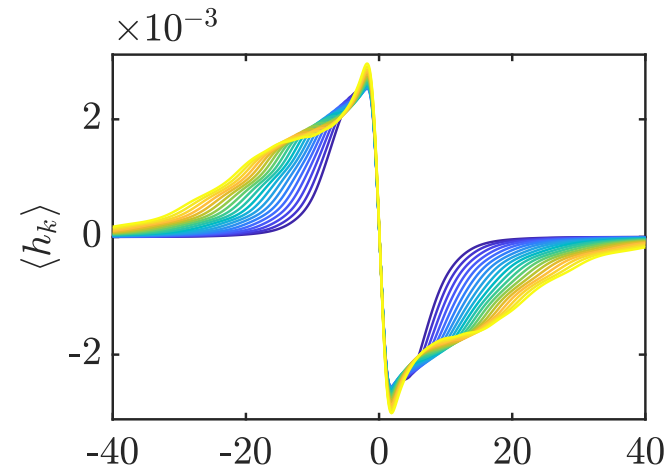

(c)

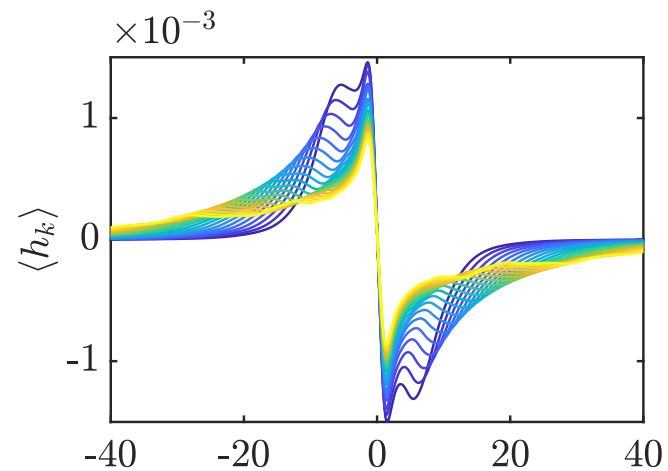

(e)

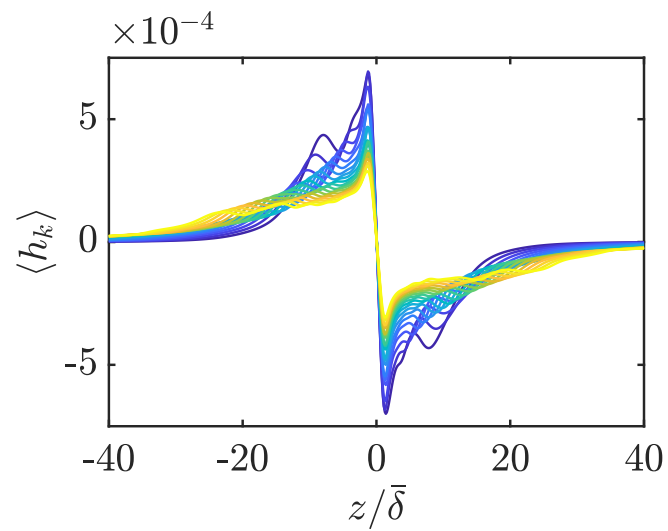

(b)

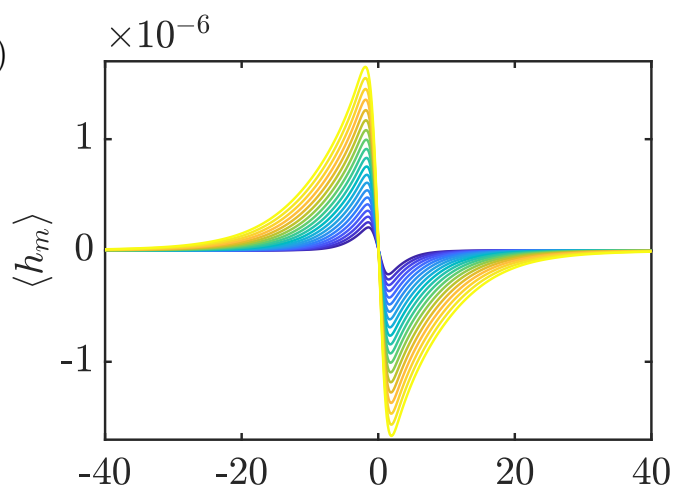

$\Omega t$

(d)

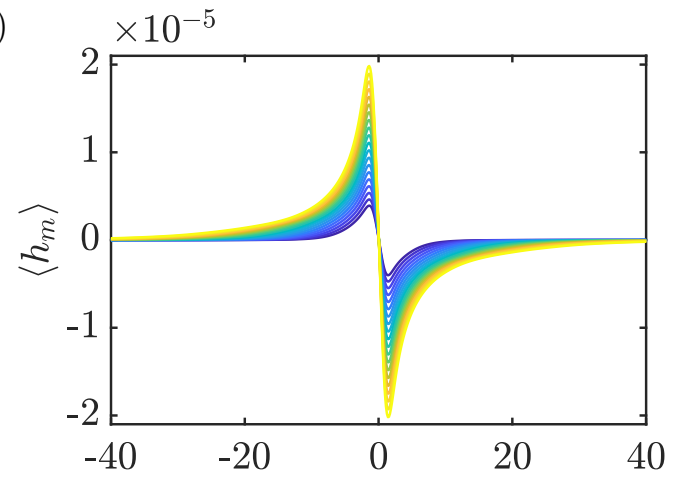

(f)

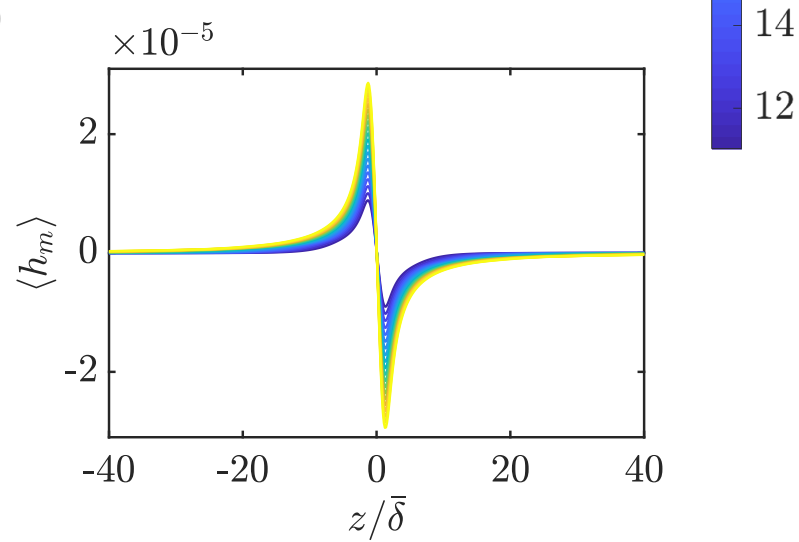

Figure 10. Helicity averaged in planes perpendicular to the rotation vector for $(a, b) S 2$, (c,d) $S 3$ and (e,f) S4. (a,c,e) Kinetic helicity and (b,d,f) magnetic helicity. The colour of the line indicates the value of $\Omega t$ specified by the legend.

ant anomalies propagate negative (positive) kinetic helicity above (below) the buoyant source, as predicted by the linear theory of inertial waves. A distribution of kinetic helicity that is negative (positive) above (below) the wave source is expected to persist for MC wave packets in the presence of a large-scale magnetic field (§2), and the magnetic helicity in the modified wave packets is predicted to be segregated in the same way. This behaviour is clear from Figures 7 and 8 . We also calculate the plane-averaged kinetic and magnetic helicity, denoted $\left\langle h_{k}\right\rangle$ and $\left\langle h_{m}\right\rangle$, where the 

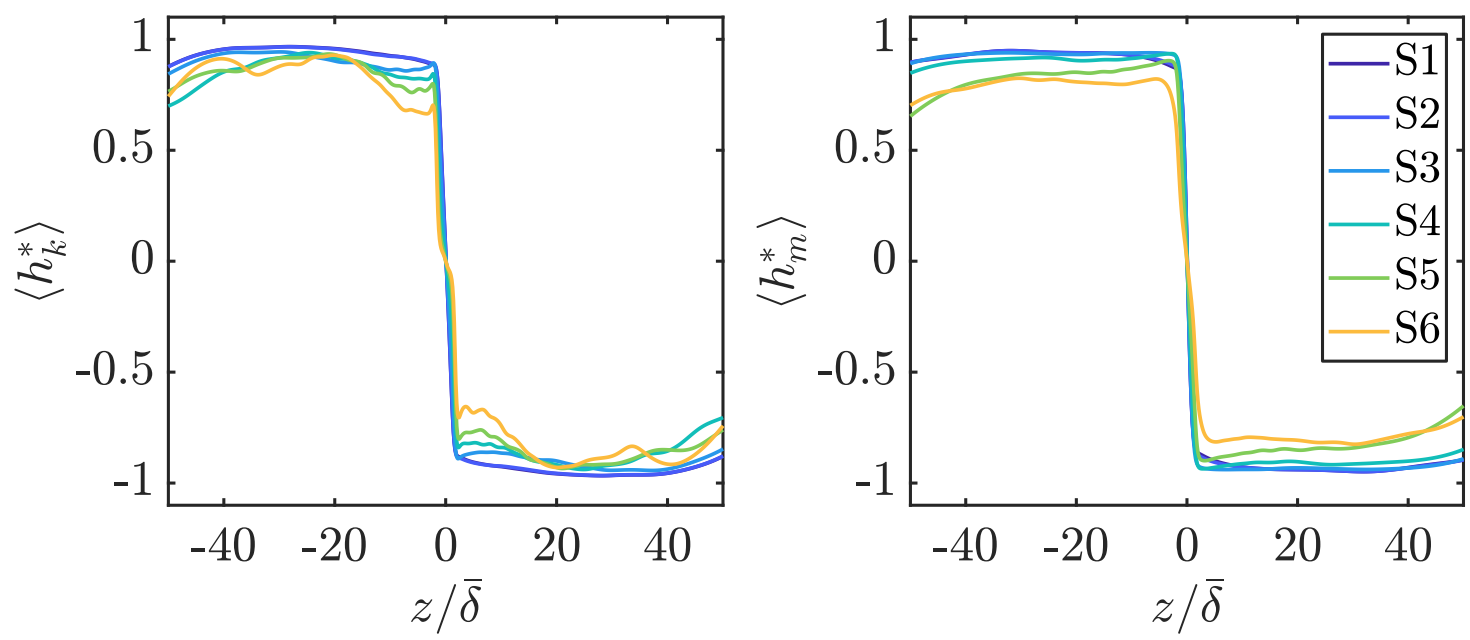

Figure 11. Relative helicity averaged in planes perpendicular to the rotation vector at $\Omega t=30$. (a) Kinetic helicity (b) magnetic helicity.

angle brackets denote the average in planes perpendicular to the rotation vector. For all the simulations, we confirm $\left\langle h_{k}\right\rangle$ and $\left\langle h_{m}\right\rangle$ are segregated so that the kinetic and magnetic helicity are negative (positive) above (below) the buoyant layer (Figure 10). The nature of the transport of helicity by the wave packets is also clear from Figure 10; helicity spreads to larger $|z|$ with time. For simulation $\mathrm{S} 2(L e=0.01)$, the kinetic helicity is greatest in magnitude, and it spreads to larger $|z|$ at a linear rate. For larger $L e$, the kinetic helicity reduces in magnitude as more kinetic energy is converted to magnetic energy, and there is enhanced Ohmic dissipation. Furthermore, the transport of helicity is more complex for S3 and S4, due to the distribution of wave-energy across different wave-types with a spectrum of axial group velocities.

A uniform magnetic field has a slightly detrimental effect on the relative kinetic and magnetic helicity transported by the wave packets. The reduction in the relative helicity is illustrated in Figure 11, where $\left\langle h_{k}^{*}\right\rangle=\left\langle h_{k}\right\rangle /\left\langle\boldsymbol{u}^{2}\right\rangle^{1 / 2}\left\langle\boldsymbol{\omega}^{2}\right\rangle^{1 / 2}$ and $\left\langle h_{m}^{*}\right\rangle=\left\langle h_{m}\right\rangle /\left\langle\boldsymbol{a}^{2}\right\rangle^{1 / 2}\left\langle\boldsymbol{b}^{2}\right\rangle^{1 / 2}$ denote the planeaveraged relative kinetic and magnetic helicity. These quantities are remarkably similar for S1 at $L e=10^{-8}$ and $\mathrm{S} 2$ at $L e=0.01$ (which is Earth-like) even at the late time shown. This observation supports our qualitative observation above, that the dynamics in S1 and S2 are alike. The curves for S2-6 display a slight decrease in both relative kinetic and magnetic helicity, indicating that these fields become less aligned with a stronger influence of the imposed magnetic field. The relative 
(a)

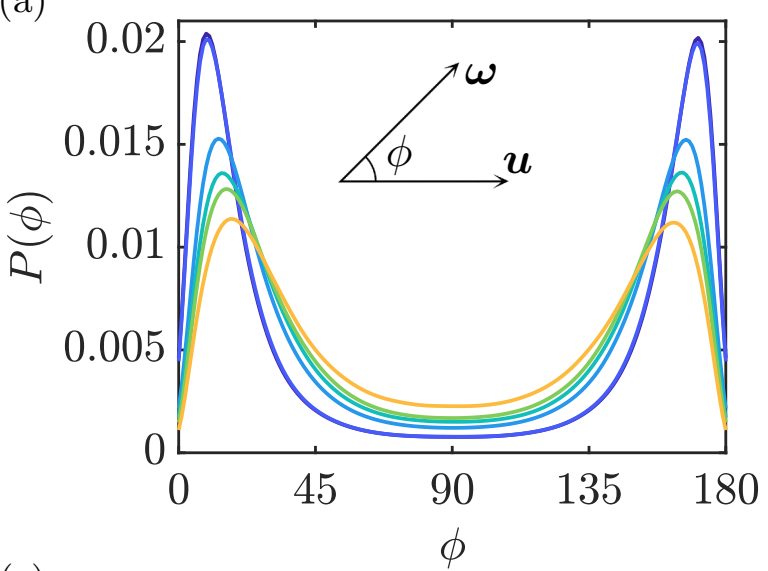

(c)

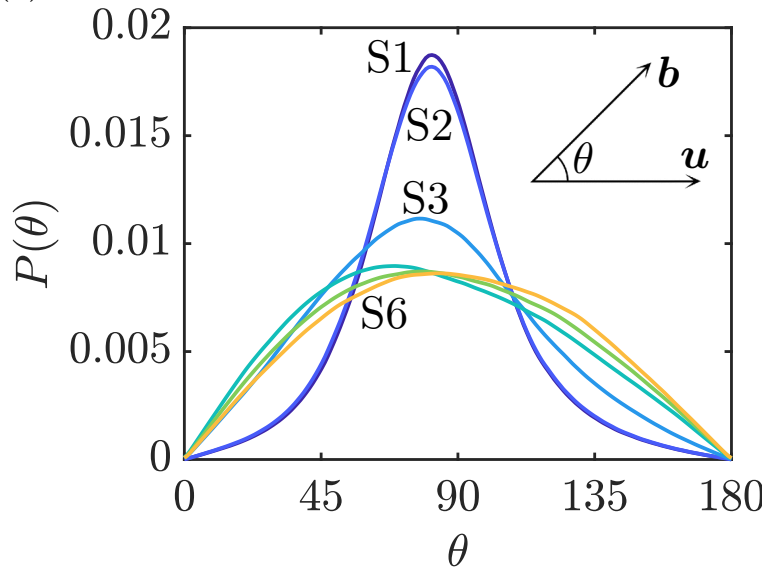

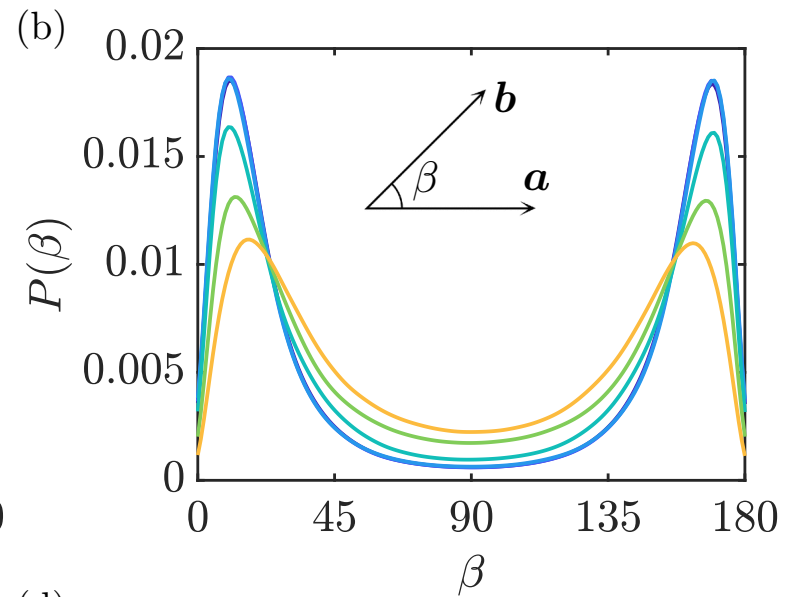

(d)

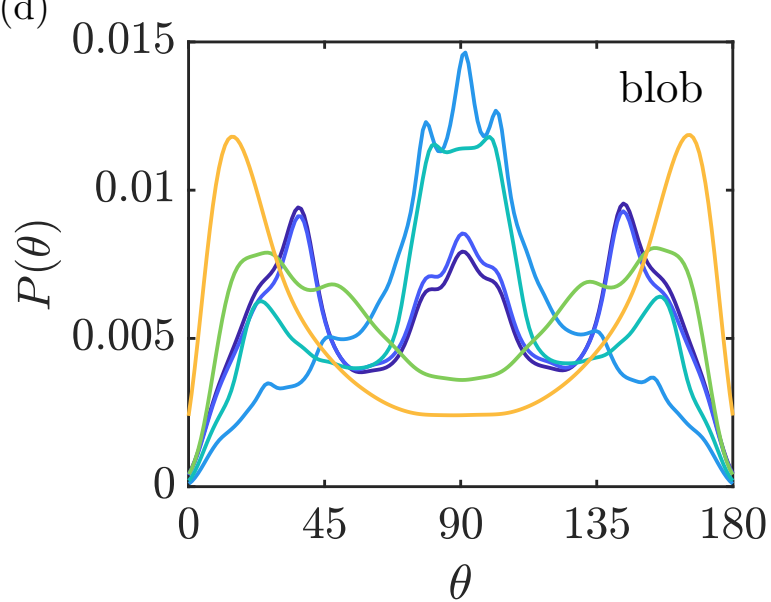

Figure 12. Approximate PDFs of the angle between vector fields at $\Omega t=30$. (a) velocity and vorticity (b) the magnetic field and its potential (c) velocity and the magnetic field. The colours are as indicated in Figure 11 and are highlighted in (c). For comparison (d) shows $P(\theta)$ for the single blob cases, three of which were discussed in $\S 3$.

helicity is closely related to the average angle between the vector fields, and this is what we will examine next.

To look at how the relative helicities vary with $L e$, we have calculated approximate probability density functions (PDFs) of the associated angles. The relative kinetic helicity $h_{k}^{*}$ is related to the angle between $\boldsymbol{u}$ and $\boldsymbol{\omega}$, say $\phi \in\left[0,180^{\circ}\right)$, such that $\phi=\cos ^{-1}\left(h_{k}^{*}\right)$. Similar relations hold for the relative magnetic helicity: $\beta=\cos ^{-1}\left(h_{m}^{*}\right)$, and the relative cross helicity: $\theta=\cos ^{-1}\left(h_{c}^{*}\right)$. The PDFs of these angles, $P(\phi), P(\beta)$ and $P(\theta)$, calculated over the wave packets, are shown in Figure 12 at $\Omega t=30$. The PDFs for S1 and (the Earth-like) S2 for $\phi, \beta$ and $\theta$ do not differ significantly. However, as Le is increased, $P(\phi)$ and $P(\beta)$ are less polarised, and the alignment between $\boldsymbol{u}$ and $\boldsymbol{\omega}$ and between $\boldsymbol{a}$ and $\boldsymbol{b}$ is marginally decreased (Figures 12a,b). The most dramatic 


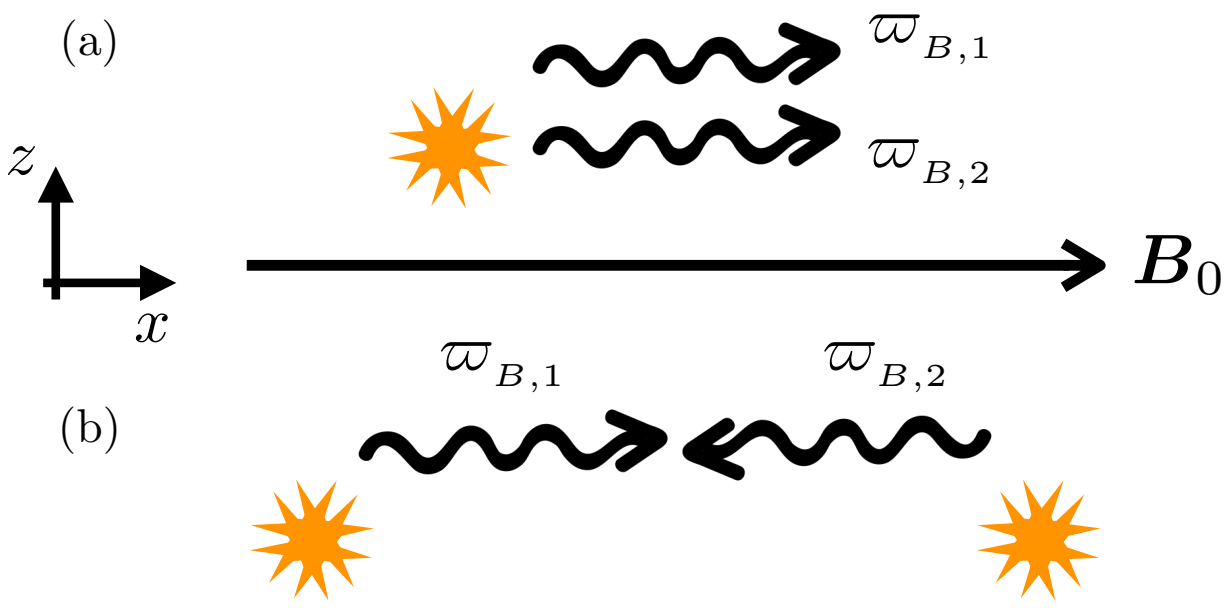

Figure 13. Two scenarios of the horizontal propagation of wave packets; wiggly arrows indicate waves. (a) Single source case and (b) two source case. In (a) wave packets propagate horizontally away from each other, and never interact. Whereas (b) shows that, with neighbouring sources, wave packets can propagate horizontally toward one another. The resulting emf of the two cases is different, see (16).

effect is in $P(\theta)$, where the PDF is quite sharply peaked for S1 and $\mathrm{S} 2$ at $\theta \approx 85^{\circ}$, indicating that $\boldsymbol{u}$ and $\boldsymbol{b}$ are nearly perpendicular throughout the waves. In the regime of low-Le: the normalised emf $\left|\mathcal{E}^{*}\right|=|\sin \theta| \sim 1$, thus the waves are very efficient at inducing an emf. For S2 and S3, at $L e=0.01$ and $L e=0.05$, which are reasonable estimates for the small scales in Earth's outer core, $P(\theta)$ has a pronounced peak near $90^{\circ}$. With a stronger mean magnetic field, the PDF is broader but still peaks near $\theta \sim 90^{\circ}$.

The alignment of the velocity and magnetic fields for the single blob case and the multiple blob (buoyant layer) case differ significantly (Figure 12c,d). With one blob there is clearly more frequent alignment between $\boldsymbol{u}$ and $\boldsymbol{b}$ than when there are wave packets emitted from multiple neighbouring blobs. For a simple explanation of this phenomenon, consider a two wave system with wave vectors $\boldsymbol{k}_{1}$ and $\boldsymbol{k}_{2}$, frequencies $\varpi_{1}$ and $\varpi_{2}$ and magnitudes $\hat{\boldsymbol{b}}_{1}$ and $\hat{\boldsymbol{b}}_{2}$,

$$
\boldsymbol{b}=\hat{\boldsymbol{b}}_{1} \cos \left(\boldsymbol{k}_{1} \cdot \boldsymbol{x}-\varpi_{1} t\right)+\hat{\boldsymbol{b}}_{2} \cos \left(\boldsymbol{k}_{2} \cdot \boldsymbol{x}-\varpi_{2} t\right)
$$

where $\left|\boldsymbol{k}_{1}\right| \approx\left|\boldsymbol{k}_{2}\right|$ and $\varpi_{1} \approx \varpi_{2}$ so that the waves are propagating information at approximately the same speed. Using (4), we can write an expression for the velocity field for the two waves

$$
\boldsymbol{u}=-\frac{\varpi_{1}}{\varpi_{B, 1}} \hat{\boldsymbol{b}}_{1} \cos \left(\boldsymbol{k}_{1} \cdot \boldsymbol{x}-\varpi_{1} t\right)-\frac{\varpi_{2}}{\varpi_{B, 2}} \hat{\boldsymbol{b}}_{2} \cos \left(\boldsymbol{k}_{2} \cdot \boldsymbol{x}-\varpi_{2} t\right)
$$

We are interested in the induced emf, which is maximal when $\boldsymbol{u}$ and $\boldsymbol{b}$ are perpendicular, so we 
expand $\boldsymbol{u} \times \boldsymbol{b}$ to find

$$
\begin{aligned}
\boldsymbol{u} \times \boldsymbol{b}= & -\frac{\varpi_{1}}{\varpi_{B, 1}}\left(\hat{\boldsymbol{b}}_{1} \times \hat{\boldsymbol{b}}_{2}\right) \cos \left(\boldsymbol{k}_{1} \cdot \boldsymbol{x}-\varpi_{1} t\right) \cos \left(\boldsymbol{k}_{2} \cdot \boldsymbol{x}-\varpi_{2} t\right) \\
& +\frac{\varpi_{2}}{\varpi_{B, 2}}\left(\hat{\boldsymbol{b}}_{1} \times \hat{\boldsymbol{b}}_{2}\right) \cos \left(\boldsymbol{k}_{1} \cdot \boldsymbol{x}-\varpi_{1} t\right) \cos \left(\boldsymbol{k}_{2} \cdot \boldsymbol{x}-\varpi_{2} t\right) .
\end{aligned}
$$

The conditions on the waves' respective wavevectors and frequencies mean the cosine terms have approximately the same argument, reducing them to $\cos ^{2}(\sim)$ terms. Averaging this expression we find

$$
\overline{\boldsymbol{u} \times \boldsymbol{b}} \approx \frac{\bar{\varpi}}{2}\left(\frac{1}{\varpi_{B, 2}}-\frac{1}{\varpi_{B, 1}}\right)\left(\hat{\boldsymbol{b}}_{1} \times \hat{\boldsymbol{b}}_{2}\right),
$$

where $\bar{\varpi}=\left(\varpi_{1}+\varpi_{2}\right) / 2$. Now consider Figure 13, which shows the two cases of wave packets propagating along the mean-field in the same direction $\left(\boldsymbol{k}_{1} \approx \boldsymbol{k}_{2}\right.$, for a single source) and toward one another $\left(\boldsymbol{k}_{1} \approx-\boldsymbol{k}_{2}\right.$, for two neighbouring sources). Evidently,

$$
\overline{\boldsymbol{u} \times \boldsymbol{b}} \approx \begin{cases}0 & \text { if } \boldsymbol{k}_{1} \approx \boldsymbol{k}_{2}, \\ \frac{\overline{\bar{m}}}{\left|\overline{\bar{\omega}}_{B}\right|}\left(\hat{\boldsymbol{b}}_{1} \times \hat{\boldsymbol{b}}_{2}\right) & \text { if } \boldsymbol{k}_{1} \approx-\boldsymbol{k}_{2},\end{cases}
$$

where $\bar{\varpi}_{B}=\left(\left|\varpi_{B, 1}\right|+\left|\varpi_{B, 2}\right|\right) / 2$. Thus, spatially overlapping wave packets which propagate along field lines toward one another are permitted to generate a significant emf in the monochromatic case, and this can only happen in the multiple blob cases. However, MC wave packets emitted from a single source propagate along the mean-field away from one another, and so never interact (see Figure 13). The above two-wave argument explains the difference between the alignment of $\boldsymbol{u}$ and $\boldsymbol{b}$ for the single blob and the multiple blob cases.

\section{IMPLICATIONS FOR DYNAMO SIMULATIONS AND PLANETARY CORES}

The $y$-averaged emf in the direction of $\boldsymbol{B}_{0}$ is well segregated positive (negative) above (below) the buoyant layer for Earth-like values of Le (Figure 7). Further, we have shown above that the environment in which there are many buoyant sources (arguably a more natural situation than a single isolated source) is beneficial to the generation of a mean emf. Now, we look at the variation of $\alpha=\mathcal{E}_{x} / B_{0}$ across the simulations with a buoyant layer. Averaging $\alpha$ over the wave packet region restricted to the top half of the box (as $\alpha$ tends to be skew-symmetric about the mid-plane) 


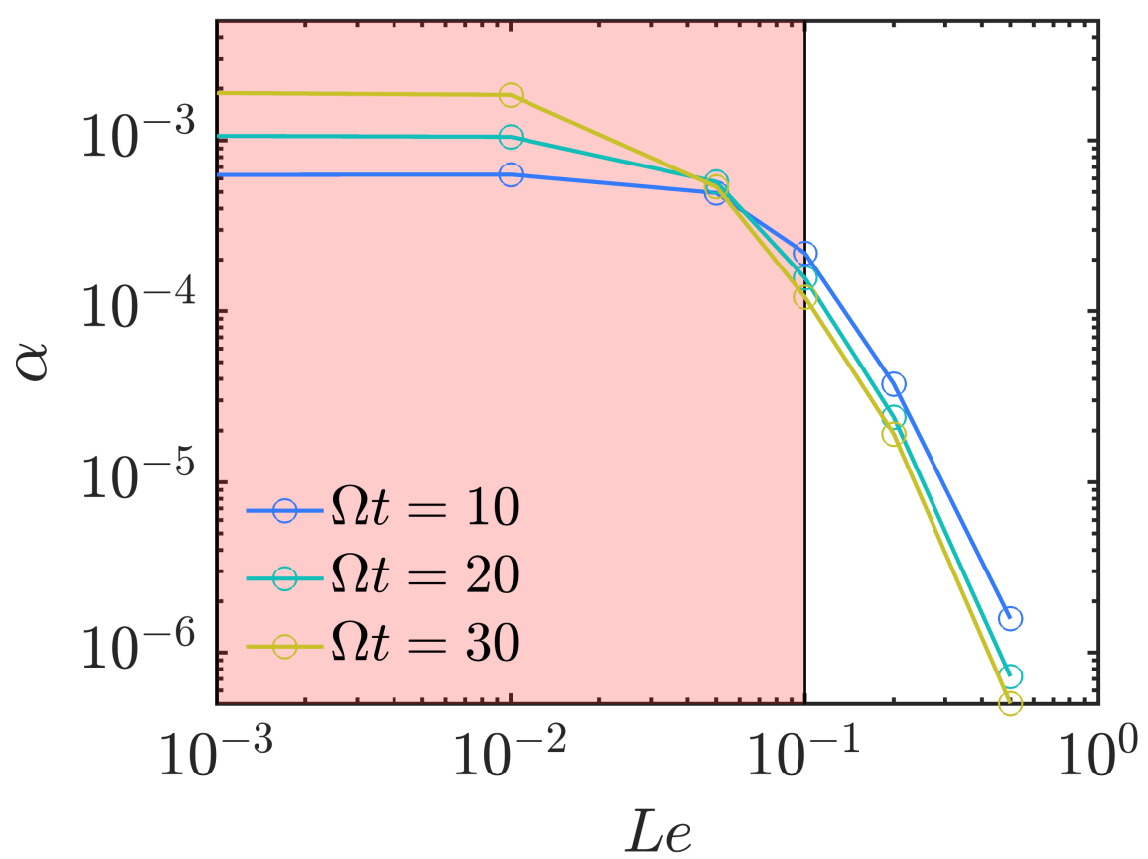

Figure 14. Average $\alpha$ for simulations S2-6. The shaded box highlights the range of Earth-like Le and the points for $\mathrm{S} 1$ ( $L e=10^{-8}$ ) have approximately the same values as for $\mathrm{S} 2$, and are omitted for clarity.

reveals that $\alpha$ remains strong for a broad range of field strengths up to $L e=0.1$; dropping to 10 $\%$ of the value for $\mathrm{S} 1$ at $L e=0.1$ (Figure 14).

The physical interpretation of the observed decrease in $\alpha$ is that as $B_{0}$ increases, lateral mixing increases between the wave packets (Figure 8). The horizontal displacement in the direction of the

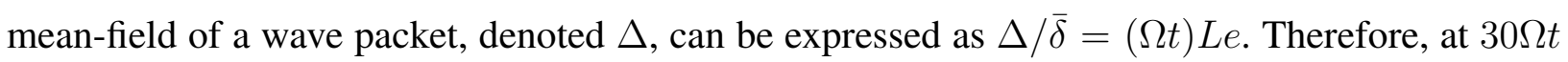
(as in Figure 8), the wave packets in S5 and S6 (at $L e=0.2,0.5$ ) have been significantly displaced. Now, the two inertial wave packets above (or indeed below) a buoyant blob have opposite signed $u_{z}$ and $\omega_{z}$ (see Figure 6). Thus, when there is a substantial amount of cross-mixing (as for $L e \geq 0.2$ ), there is cancellation in the components of the velocity field in the overlapping region, and the magnitude of the velocity is reduced. This is also true for MC wave packets, and the resulting decrease in the velocity and magnetic field perturbations is consistent with the observed reduction in $\alpha$ (Figure 14).

It is also interesting to take a closer look at the PDF of $\theta$, the angle between $\boldsymbol{u}$ and $\boldsymbol{b}$ defined in $\S 4$ (Figure 12c). Evidently we have a strong emf for $|\cos \theta| \approx 0$ and $|\sin \theta| \approx 1$, which is the most likely scenario for simulations S1-6. For S1 and S2 (at $L e=10^{-8}, 0.01$ ), the peak near 
$\theta \sim 90^{\circ}$ indicates a strong induced emf. For $\mathrm{S} 4-6,|\cos \theta| \approx 0$ and $|\sin \theta| \approx 1$ are still the most pronounced parts of the PDF, but these values are approximately half as likely as for S1 and S2. These observations are independent of the magnitude of the kinetic energy, unlike the explanation above.

Recent numerical dynamo simulations have highlighted the presence of rapid time-scale inertial and hydromagnetic waves (Schaeffer et al. 2017; Ranjan et al. 2018; Aubert 2019). Aubert (2019) observes "quasi-geostrophic Alfvén waves" propagating along the cylindrical radial magnetic field, launched from a buoyant plume. The description of these fast time-scale, localised events is very reminiscent of the wave packets observed in our simulations (and in Bardsley \& Davidson 2016). The convective structures outside the tangent cylinder in the extreme simulation of Schaeffer et al. (2017) are characterised by Lehnert numbers in the range $0.01-0.1$. The flow is chaotic in space and time, quasi-geostrophic, and highly helical — fast time-scale MC wave packets like those observed in Figures $7 \& 8(\mathrm{~S} 2-4)$ are good candidates for the maintenance of these flow structures. As planetary dynamo simulations progress to increasingly turbulent states, it is expected that the propagation of wave packets will play an ever more important role in the dynamics, and possibly the dynamo mechanism (Davidson \& Ranjan 2015).

The magnetic field inside the core will certainly be heterogeneous, with the possibility of having regions of the core where the magnetic field is much weaker/stronger than the average. The more dipolar numerical simulations often exhibit an azimuthal mean-field which has opposite signs in each hemisphere, which is relatively weak outside of the tangent cylinder, and goes to zero at the equator (Roberts \& King 2013; Schaeffer et al. 2017). This type of anti-symmetric meanfield would change our results in two main ways. The helicity distribution would remain negative (positive) above (below) the source. However the induced emf would now be positive both above and below, as required for an $\alpha^{2}$ dynamo. Second, our results suggest that a weaker azimuthal field in the regions of the equatorial plane would be beneficial for the generation and propagation of kinetic helicity and the emf, although the dispersion relation of a wave packet may evolve along its path (Bardsley \& Davidson 2017). As the wave packets enter regions of stronger field at 
higher latitudes, they would be subject to enhanced Ohmic dissipation, reducing the likelihood of cancellation by reflections at the core-mantle boundary.

The picture outlined above is consistent with the cartoon outlined in Figure 1 for an $\alpha^{2}$ dynamo driven by helical convection/waves. Using ray theory, Bardsley \& Davidson (2017) deduced the effect of an axially varying anti-symmetric mean-field on MC wave dispersion. They concluded that even though IA waves are de-focused by the slow variation of $B_{0}$, the fact that $L e$ is small in Earth's core implies that the intermediate MC waves disperse more or less axially, and this is what we observe for a sea of MC wave packets for $L e<0.1$. The time-scales $\tau_{\Omega}, \tau_{A}$ and $\tau_{u}=\ell / u$ are ordered such that $30 \tau_{\Omega} \lesssim \tau_{A} \ll \tau_{u}$, which suggests that inertial/MC wave packets continually launched from a chaotic buoyancy field would be particularly effective at maintaining elongated flow structures and driving an $\alpha$-effect.

In Jault (2008), the axisymmetric motions launched by a jerk of the inner sphere, which travel rapidly along the rotation axis and migrate outwards at the radial Alfvén speed are certainly similar to the propagation of IA wave packets (see also the recent experiments by Tigrine et al. 2019). It has been noted by Jault (2008) that numerical dynamos with large-scale Lehnert numbers less than $\sim 3 \times 10^{-2}$ are broadly quasi-geostrophic. Using the average mean spherical harmonic degree in the kinetic energy spectrum of the dynamos in the dataset of Christensen \& Aubert (2006), this translates to a 'local' Lehnert number of $\sim 0.2$. This is consistent with the isosurfaces presented in Figures $7 \& 8$ - the flow is reasonably columnar in S4 and becomes less columnar in S5-6.

In recent (more turbulent) geodynamo simulations (Schaeffer et al. 2017; Aubert 2019), buoyant anomalies outside the tangent cylinder have the potential to launch wave packets like those observed here. Ranjan et al. (2018) showed that the time derivative of axial velocity is a good indicator for localised wave-fronts, we corroborate this finding. This approach could be combined with analysis of the local energy density ratio and relative helicities to search for MC wave packets emitted from buoyant blobs/plumes in dynamo simulations. 


\section{CONCLUDING REMARKS}

Magnetic-Coriolis wave packets which propagate through a uniform transverse mean-field generate a significant mean emf at Earth-like values of the Lehnert number. Further, mean emf remains well segregated positive (negative) above (below) the wave source for Lehnert numbers smaller than 0.1. For mean-fields with a larger magnitude the induced emf is reduced, and the $\alpha$-effect is less influential. The wave packets in our simulations that transport a high energy density are well described by three groups: weakly modified inertial $\left(c_{g z} \sim 2 \Omega / k\right)$, inertial-Alfvén $\left(c_{g z} \sim \Omega / k\right.$, $\boldsymbol{k} \cdot \boldsymbol{\Omega} \approx 0$ ) and intermediate magnetic-Coriolis wave packets (fast and columnar at low-Le). Our simulations cannot run for sufficiently many rotation times for the signature of magnetostrophic waves to become distinct.

For Earth-like values of the Lehnert number, we find that on time-scales of the order of weeks:

i The wave packets are coherent and columnar (Figure 7).

ii The velocity and magnetic fields are mostly perpendicular, and the wave packets induce a sizeable emf, which is greater when there are multiple neighbouring sources.

iii Magnetic-Coriolis wave packets generate a spatially organised $\alpha$-effect, where the average $\alpha$ remains strong for a broad range of field strengths up to $L e=0.1$.

iv The horizontally averaged $\mathcal{E}_{x}$ is well correlated with $-\left\langle h_{k}\right\rangle$.

The helical wave dynamo model of Davidson (2014) requires kinetic helicity that is sufficiently intense, and that is distributed negative (positive) in the north (south) outside the tangent cylinder (Figure 1). The model suggests that helical wave packets launched from an equatorially biased heat-flux could set up an $\alpha^{2}$ dynamo cycle as described in Figure 1, where the velocity field principally acts on the horizontal components of the mean-field. However, this model does not explicitly take into account the dynamic effect of a mean magnetic field. The work presented here suggests that such a model is indeed feasible in Earth's core, even for upper estimates of the toroidal field strength. We note that our results are essentially the same if we model the local cylindrical radial field $B_{r}$ as $B_{0} \boldsymbol{e}_{y}$, yielding an $\alpha_{y y}$. 


\section{ACKNOWLEDGMENTS}

The authors acknowledge interesting discussions on magnetic-Coriolis waves with Oli Bardsley. $\mathrm{PD}$ and $\mathrm{BM}$ conceived and designed the analysis. BM performed the analysis and wrote the paper. PD provided valuable insights and interpretations and contributed to the manuscript. This work was completed during a doctoral studentship sponsored by the Leverhulme trust UK, grant no. RPG-2015-195/RG77943.

This paper has been produced using the Blackwell Scientific Publications GJI LTEX2e class file.

\section{REFERENCES}

Aubert, J., 2019. Approaching Earth's core conditions in high-resolution geodynamo simulations, Geophysical Journal International, 219(1), 137-151.

Bardsley, O. P. \& Davidson, P. A., 2016. Inertial-Alfvén waves as columnar helices in planetary cores, Journal of Fluid Mechanics, 805, R2.

Bardsley, O. P. \& Davidson, P. A., 2017. The dispersion of magnetic-Coriolis waves in planetary cores, Geophysical Journal International, 210(1), 18-26.

Christensen, U. R. \& Aubert, J., 2006. Scaling properties of convection-driven dynamos in rotating spherical shells and application to planetary magnetic fields, Geophysical Journal International, 166(1), 97114.

Davidson, P. A., 2005. Turbulence : an introduction for scientists and engineers, Oxford University Press.

Davidson, P. A., 2013. Scaling laws for planetary dynamos, Geophysical Journal International, 195(1), $67-74$.

Davidson, P. A., 2014. The dynamics and scaling laws of planetary dynamos driven by inertial waves, Geophysical Journal International, 198(3), 1832-1847.

Davidson, P. A. \& Ranjan, A., 2015. Planetary dynamos driven by helical waves-II, Geophysical Journal International, 202(3), 1646-1662.

Davidson, P. A., Staplehurst, P. J., \& Dalziel, S. B., 2006. On the evolution of eddies in a rapidly rotating system, Journal of Fluid Mechanics, 557, 135-144.

Gillet, N., Jault, D., Canet, E., \& Fournier, A., 2010. Fast torsional waves and strong magnetic field within the Earth's core, Nature, 465, 74-77.

Gómez, D. O., Mininni, P. D., \& Dmitruk, P., 2005. Parallel simulations in turbulent MHD, Physica Scripta, 2005(T116), 123.

Guervilly, C., Cardin, P., \& Schaeffer, N., 2019. Turbulent convective length scale in planetary cores, Nature, 570(7761), 368-371. 
Hide, R., 1966. Free hydromagnetic oscillations of the Earth's core and the theory of the geomagnetic secular variation, Philosophical Transactions of the Royal Society of London. Series A, Mathematical and Physical Sciences, 259(1107), 615-647.

Jault, D., 2008. Axial invariance of rapidly varying diffusionless motions in the Earth's core interior, Physics of the Earth and Planetary Interiors, 166(1-2), 67-76.

Malkus, W. V. R., 1967. Hydromagnetic planetary waves, Journal of Fluid Mechanics, 28(4), 793-802.

McDermott, B. R. \& Davidson, P. A., 2019. A physical conjecture for the dipolar-multipolar dynamo transition, Journal of Fluid Mechanics, 874, 995-1020.

Moffatt, H. K., 1970. Dynamo action associated with random inertial waves in a rotating conducting fluid, Journal of Fluid Mechanics, 44(4), 705-719.

Moffatt, H. K., 1978. Magnetic field generation in electrically conducting fluids, Cambridge University Press.

Olson, P., Christensen, U. R., \& Glatzmaier, G. A., 1999. Numerical modeling of the geodynamo: mechanisms of field generation and equilibration, Journal of Geophysical Research: Solid Earth, 104(B5), 10383-10404.

Ranjan, A., Davidson, P. A., Christensen, U. R., \& Wicht, J., 2018. Internally driven inertial waves in geodynamo simulations, Geophysical Journal International, 213(2), 1281-1295.

Roberts, P. H. \& King, E. M., 2013. On the genesis of the Earth's magnetism, Reports on Progress in Physics, 76(9), 096801.

Sakuraba, A. \& Roberts, P. H., 2009. Generation of a strong magnetic field using uniform heat flux at the surface of the core, Nature Geoscience, 2(11), 802-805.

Schaeffer, N., Jault, D., Nataf, H.-C., \& Fournier, A., 2017. Turbulent geodynamo simulations: a leap towards Earth's core, Geophysical Journal International, 211(1), 1-29.

Tigrine, Z., Nataf, H.-C., Schaeffer, N., Cardin, P., \& Plunian, F., 2019. Torsional Alfvén waves in a dipolar magnetic field: experiments and simulations, Geophysical Journal International, 219(1), S83-S100. 